\title{
Local CSI Based Full Diversity Achieving Relay Selection for Amplify-and-Forward Cooperative Systems
}

\author{
Batu K. Chalise, Senior Member, IEEE, Yimin D. Zhang, Senior Member, IEEE, and Moeness G. Amin, Fellow, IEEE
}

\begin{abstract}
This paper proposes a new relay selection method based on local channel state information (CSI) for a system consisting of a source, a destination and an arbitrary number of amplify-and-forward relay nodes. A set of candidate relays, whose source-relay (S-R) links are not in outage, is formed. An S-R link is in outage if its signal-to-noise ratio (SNR) is below the predefined threshold value of the destination SNR plus some adjustable margin. The candidate relay that yields the maximum second-hop SNR is then selected at the destination. Unlike the opportunistic relaying (OR) based on full CSI (F-CSI), the proposed method requires to equip the destination node with the instantaneous CSI of the S-R channel corresponding to only the selected relay. It is qualitatively shown that the training overhead of the proposed relay selection is less than or comparable to that of the partial relay selection (PRS) but can be much smaller than that of the OR with F-CSI. However, the proposed scheme requires to estimate a higher number of single-input single-output (SISO) channels than in the PRS scheme. The exact and asymptotic expressions of outage probability are derived and it is shown that the proposed method achieves full diversity. Simulation results verify theoretical analysis, and show that, for the optimized margin, the proposed scheme provides performance which is comparable to the method having F-CSI. The results also show that the proposed method significantly outperforms PRS, which may justify the linear order of the computational complexity associated with the estimation of the additional SISO channels and the low-cost one-dimensional line search used for optimizing the margin.
\end{abstract}

Index Terms-Amplify-and-forward relay, cooperative communications, diversity gain, outage probability, relay selection.

\section{INTRODUCTION}

$\mathbf{C}$ OOPERATIVE communication [1], [2] is an emerging technique that improves spatial diversity ${ }^{1}$, coverage range and system throughput in cellular communications [4].

Manuscript received November 15, 2012; revised April 24, 2013 and July 09, 2013; accepted July 18, 2013. Date of publication July 26, 2013; date of current version September 17, 2013. The associate editor coordinating the review of this manuscript and approving it for publication was Dr. Tongtong Li. Part of this work was presented at IEEE Globecom 2012, Anaheim, CA, USA, December 2012.

B.K. Chalise was with the Center for Advanced Communications, Villanova University, Villanova, PA 19085 USA. He is now with ArrayComm, LLC, San Jose, CA 95110 USA (e-mail: chalise@arraycomm.com)

Y.D. Zhang and M.G. Amin are with the Center for Advanced Communications, Villanova University, Villanova, PA 19085 USA (e-mail: yimin.zhang@villanova.edu; moeness.amin@villanova.edu).

Color versions of one or more of the figures in this paper are available online at http://ieeexplore.ieee.org.

Digital Object Identifier 10.1109/TSP.2013.2274958

${ }^{1}$ Diversity is defined as the slope of the error probability or outage probability curve in log-scale at the high signal-to-noise ratio (SNR) regimes [3].
A key component of cooperation is relaying in which a source (base station) seeks the assistance of user terminals (dedicated relay terminals) in its coverage area to relay the source signal to the destination (user equipment). Among different relay protocols [2], the amplify-and-forward (AF) protocol is often used due to its simplicity. However, in systems with multiple relays, the feedback requirements and the overall implementation become complicated and can prove costly. System complexity and cost rise in multi-antenna cooperative systems that employ beamformers over the set of nodes (source, relays, destinations) [5], [6]. In this context, relay selection is key to reducing signaling overhead and system design complexity while maintaining the diversity gain [7]-[9].

The relay selection method for AF relays is often based on the end-to-end SNR, which is commonly referred to as opportunistic relaying $(O R)$ in the literature [8], [9]. The authors of [7] proposed a timer based distributed approach for implementing $\mathrm{OR}$, where each relay node estimates its source-relay (S-R) and relay-destination (R-D) channels by listening to the request-tosend and clear-to-send packets exchanged between the source and destination, respectively. As stated in [7], this approach requires some level of communication between the relay nodes, either directly or through the source or the destination, and explicit timing synchronization between the source and destination if there is no direct link between them. An alternative way for implementing OR is by means of a centralized method [8], [9], where the relay selection algorithm is executed at the destination node assuming that it has global channel state information (CSI), i.e., CSI of all R-D and S-R channels. However, this requires the relays to estimate the S-R channels and the destination to estimate the R-D channels. It follows that the relay nodes must then inform the destination about the CSI of all S-R channels ${ }^{2}$. This, in turn, reduces the bandwidth efficiency and increases power consumption. For essence, in the centralized OR, also referred to as full CSI (F-CSI) method, non-local CSI at the central node where the relay selection algorithm is executed, is necessary. With the availability of full CSI, OR provides full diversity gain and the overall optimal performance.

On the other hand, a simple relay selection method [12] can be implemented without the stringent requirement of global CSI

\footnotetext{
${ }^{2}$ For applications that do not involve relay selection, the challenges of channel estimation (CE) in AF relay systems have been highlighted in [10] and [11], where the overall source-relay-destination (S-R-D) channel is estimated for limiting the training overhead. However, since the overall channel does not exactly reflect the end-to-end SNR, the OR built upon such overall CE can be easily suboptimal, and thus, separate estimations of the S-R and R-D channels are considered.
} 
at the central node. This method, known as partial relay selection (PRS), requires only knowledge of the first-hop (i.e., S-R) channels for selecting the best relay [12]. Therefore, PRS reduces feedback requirements for acquiring CSI, making it attractive, especially for large multihop networks in fast fading channel environments. In [12]-[13] and [14], the performance of the PRS scheme is analyzed, respectively, for CSI-assisted and fixed-gain AF relaying systems. However, it can be seen from these works that the PRS method does not achieve full diversity. In particular, irrespective of the number of relays, the diversity order of the PRS scheme is limited to only one. In our recent work [15], it is shown that the PRS scheme for multi-antenna cooperative systems with beamforming also suffers diversity loss for general antenna configurations.

This paper proposes a novel relay selection scheme with an improved tradeoff between complexity and performance for the AF protocol based cooperative systems. A key feature of this selection is relying on the local CSI, where the relay is selected at the destination under the condition that the relays and destination only have their respective instantaneous receive CSI, i.e., the relays have CSI of the S-R channels and the destination has CSI of the R-D channels. Furthermore, the destination does not require CSI of its non-local channels, i.e., the S-R channels, for making a decision on the best relay ${ }^{3}$. In the proposed method, the relay that gives the maximum second-hop SNR is selected at the destination, from only a subset of relays (candidate relays) for which the S-R links are not in outage. An S-R link is in outage if the corresponding link SNR is below the threshold value set for determining the outage event of the end-to-end transmission plus some adjustable margin. It is important to emphasize that a similar concept, i.e., the concept of selecting the best relay at the destination from a subset of relays which can successfully decode the source signal is known as selection cooperation (SC) in decode-and-forward (DF) protocols (see [17], [18] for Rayleigh fading and [19] for Nakagami- $m$ fading channels). However, unlike in the DF systems, the destination SNR in the AF relaying is a function of SNRs of both first and second hops corresponding to the candidate relays. Therefore, in contrast to the DF systems, relay selection based on CSI of only the R-D channels of the candidate relays is not necessarily optimal for our case. Towards this end, we propose a novel concept of introducing an adjustable margin and a method for optimizing it. It is also interesting to note that the proposed method with no margin can be viewed as a direct application of SC for the AF relays, which is shown to achieve no full diversity gain in general. To the best of our knowledge, this finding itself is new since the performance of $\mathrm{SC}$ in $\mathrm{AF}$ relays is not known from previous studies. Moreover, the statistics of the destination SNR in the proposed scheme differ significantly from those in the DF protocols, meaning that a different approach is required for the performance analysis of the proposed method.

The training/signaling and communication overhead ${ }^{4}$, and the computational complexity of $\mathrm{CE}$ of the proposed selection is qualitatively compared with the PRS and F-CSI methods.

${ }^{3} \mathrm{CSI}$ of the S-R channel corresponding to only the selected relay is forwarded to the destination for decoding source signal.

${ }^{4}$ This refers to an overhead required for broadcasting the index (w.r.t. the set of all relays) of the selected relay (cf. Section II.B).
All three methods are found to have the same communication overhead. It is demonstrated that the training overhead of the proposed method is less than or comparable to that of the PRS method but can be much smaller than that of the F-CSI method. However, the proposed scheme requires to estimate a higher number of single-input single-output (SISO) channels than the PRS scheme. The complexity of estimating a SISO channel is of only linear order (w.r.t. the length of a training signal) and can become further less significant for slowly time-varying block-fading channels [20], [21]. Nonetheless, the complexity of additional SISO CEs and the complexity associated with the optimization of the margin, which requires low-cost one-dimensional line search (cf. Section IV.A), may be viewed as a price worth paying for large performance gains over the PRS method.

We derive exact expressions for the outage probability of the proposed method, which are utilized to provide the asymptotic expressions for evaluating the diversity orders. The asymptotic expressions are then employed for optimizing the aforementioned margin. It is shown that the proposed scheme achieves full diversity order. Simulation results demonstrate that the proposed method significantly outperforms PRS and provides performance comparable to that of the F-CSI [13] for the optimized margin. The theoretical results of the proposed method are obtained assuming that both the S-R and R-D channels are identically distributed. However, it will be evident from the derivations that the analysis can be easily extended to the case with non-identically distributed channels. Since the proposed relay selection scheme is different from the techniques in [6]-[9], [12]-[15] and [17]-[20], and we need to deal with the joint probabilities of statistically dependent random variables, these existing techniques are neither directly applicable nor easily extendable to our problem. A new and rigorous approach is proposed for handling these joint probability terms. For the asymptotic results corresponding to zero margin, a more extensive series expansion [22] is employed for the first order modified Bessel function of the second type since the standard expansion (e.g., in [20]) does not enable us to obtain the diversity order. When the margin is larger than zero, we propose to utilize the series expansion (logarithmic case) of the Whittaker hypergeometric functions [22] for obtaining the diversity order. This approach, to the best of our knowledge, has not been reported before in the technical literature.

The rest of the paper is organized as follows. The system model, the proposed relay selection protocol, and a qualitative assessment of the training overhead and computational complexity are presented in Section II. The exact expressions for the outage probability are derived in Section III, whereas in Section IV, the corresponding asymptotic expressions are derived. The method for optimizing the margin is also provided in Section IV. In Section V, the performance of the proposed method is compared with the PRS and F-CSI methods. Section VI concludes the paper.

Notations: $f_{\alpha}(x), \quad \operatorname{Pr}\{\cdot\}, \quad \operatorname{Pr}\left\{x_{1}, x_{2}\right\}, \quad \operatorname{Pr}\left\{\left[x_{1} \cup x_{2}\right]\right\}$, $\operatorname{Pr}\left\{x_{1} \mid x_{2}\right\}$, and $\mathcal{O}(x)$ denote probability density function (PDF) of a continuous random variable $\alpha$, the probability operator, the probability of the intersection between $x_{1}$ and $x_{2}$, 
the probability of the union of $x_{1}$ and $x_{2}$, the probability of $x_{1}$ conditioned to $x_{2}$, and the higher order terms of $x$, respectively.

\section{System Model and Proposed Relay Selection}

We examine a cooperative network that consists of a source, a destination and $R \geq 1$ distributed relay nodes, all equipped with a single antenna. The relays operate in a half-duplex mode and employ the AF protocol. All channels are assumed to be slowly time-varying, i.e., the channel coherence time is much larger than the symbol/block duration. Let $h_{\mathrm{s}, j}$ and $h_{j, \mathrm{~d}}$ denote the complex channel coefficients between the source and the $j$ th relay $(j=1, \ldots, R)$, and between the $j$ th relay and the destination, respectively. Each node (source and relay) transmits with a given power. Let $P_{\mathrm{S}}$ denote the source power and $P_{\mathrm{R}, j}$ the transmit power of the $j$ th relay node. The path gains for the S-R and R-D channels are denoted by $c_{\mathrm{s}, j}$ and $c_{j, \mathrm{~d}}$, respectively. We consider Rayleigh fading environment, i.e., $h_{\mathrm{s}, j}$ and $h_{j, \mathrm{~d}}$ are assumed to be zero-mean circularly symmetric complex Gaussian (ZMCSCG) random variables with unit variance. The direct link between the source and destination does not exist, i.e., the destination is out of the coverage range of the source node. All communications, i.e., communications between the source and relays and those between the relays and destination take place in the same frequency band.

The signal received by the $j$ th relay in the first phase of signal transmission is given by

$$
y_{\mathrm{r}, j}=\sqrt{P_{\mathrm{s}} c_{\mathrm{s}, j}} h_{\mathrm{s}, j} x+n_{\mathrm{r}, j}
$$

where $x$ is the source signal with zero-mean and unit variance and $n_{\mathrm{r}, j}$ is the relay noise described by a ZMCSCG random variable with variance $\sigma_{\mathrm{r}}^{2}$. The $j$ th AF relay normalizes the received signal and forwards the following signal to the destination:

$$
\tilde{y}_{\mathrm{r}, j}=\frac{\sqrt{P_{\mathrm{r}}}\left[\sqrt{P_{\mathrm{s}} c_{\mathrm{s}, j}} h_{\mathrm{s}, j} x+n_{\mathrm{r}, j}\right]}{\sqrt{P_{\mathrm{s}} c_{\mathrm{s}, j}\left|h_{\mathrm{s}, j}\right|^{2}+\sigma_{\mathrm{r}}^{2}}} .
$$

The signal received by the destination in the second phase of transmission from the $j$ th relay is

$$
\tilde{y}_{\mathrm{d}, j}=\frac{\sqrt{P_{\mathrm{r}} c_{j, \mathrm{~d}}} h_{j, \mathrm{~d}}\left[\sqrt{P_{\mathrm{s}} c_{\mathrm{s}, j}} h_{\mathrm{s}, j} x+n_{\mathrm{r}, j}\right]}{\sqrt{P_{\mathrm{s}} c_{\mathrm{s}, j}\left|h_{\mathrm{s}, j}\right|^{2}+\sigma_{\mathrm{r}}^{2}}}+n_{\mathrm{d}, j}
$$

where $n_{\mathrm{d}, j}$ is the noise at the destination, which is assumed to be a ZMCSCG random variable with variance $\sigma_{\mathrm{d}}^{2}$. The SNR at the destination due to the $j$ th relay can be expressed as

$$
\begin{aligned}
\gamma_{j}=\frac{P_{\mathrm{s}} P_{\mathrm{r}} c_{\mathrm{s}, j} c_{j, \mathrm{~d}}\left|h_{\mathrm{s}, j}\right|^{2}\left|h_{j, \mathrm{~d}}\right|^{2}}{P_{\mathrm{r}} c_{j, \mathrm{~d}}\left|h_{j, \mathrm{~d}}\right|^{2} \sigma_{\mathrm{r}}^{2}+P_{\mathrm{s}} c_{\mathrm{s}, j}\left|h_{\mathrm{s}, j}\right|^{2} \sigma_{\mathrm{d}}^{2}+\sigma_{\mathrm{r}}^{2} \sigma_{\mathrm{d}}^{2}} \\
\triangleq \frac{f_{j} \alpha_{\mathrm{s}, j} g_{j} \alpha_{j, \mathrm{~d}}}{g_{j} \alpha_{j, \mathrm{~d}}+f_{j} \alpha_{\mathrm{s}, j}+1}
\end{aligned}
$$

where $\alpha_{\mathrm{s}, j}=\left|h_{\mathrm{s}, j}\right|^{2}, \alpha_{j, \mathrm{~d}}=\left|h_{j, \mathrm{~d}}\right|^{2}, f_{j} \triangleq \frac{P_{\mathrm{s}} \cdot c_{\mathrm{s}, j}}{\sigma_{\mathrm{r}}^{2}}$ and $g_{j} \triangleq$ $\frac{P_{\mathrm{r}, j} \cdot c_{j, \mathrm{~d}}}{\sigma_{\mathrm{d}}^{2}}$ denote the average SNRs of the $j$ th S-R and R-D links, respectively. Since $h_{\mathrm{s}, j}$ and $h_{j, \mathrm{~d}}$ are ZMCSCG random variables, $\alpha_{\mathrm{s}, j}$ and $\alpha_{j, \mathrm{~d}}$ are exponentially distributed.

In the sequel, we develop a relay selection method which assumes that $\gamma_{j}$ is not known to the destination (or any other nodes), meaning that the destination does not have CSI of the $\mathrm{S}-\mathrm{R}$ channels for determining the best relay. However, after the relay is selected, it sends CSI of the corresponding S-R channel to the destination for decoding the source signal optimally. This means that $\gamma_{j}$ corresponding to only the selected relay (e.g., $\gamma_{q}$, where $q$ is the index of the selected relay) is known to the destination in each channel coherence time. Consequently, such $\gamma_{q}$ is used for the outage probability analysis.

\section{A. Protocol Description}

The proposed protocol is motivated from a simple fact related to the upper bound of $\gamma_{j}$. Let $\gamma_{\mathrm{th}}$ be the threshold value of the destination SNR for determining the outage probability at the destination. Since $\gamma_{j} \leq \min \left(f_{j} \alpha_{\mathrm{s}, j}, g_{j} \alpha_{j, \mathrm{~d}}\right)$ [23], it is clear that if $f_{j} \alpha_{\mathrm{s}, j}<\gamma_{\mathrm{th}}$, the $j$ th end-to-end transmission from the source to the destination is certain to be in outage, irrespective of the values of $g_{j} \alpha_{j, \mathrm{~d}}$. Taking this fact into account, we proceed to formally describe the protocol as follows. In the proposed scheme, the destination node selects the best relay during a training phase. The source node broadcasts a training signal and each relay determines whether it is in outage, i.e., the SNR of its link with the source is below $\gamma_{\text {th }}$ plus some margin. The motivation for using the margin will be clarified later. Let $\mathcal{A}$ be a set of relays (candidate relays) with their S-R links not in outage. The candidate relay that gives the maximum R-D SNR is then selected at the destination. The training method required for implementing the proposed selection will be described in details in the following subsection. The set $\mathcal{A}$ can be expressed as

$$
\mathcal{A}=\left\{j, \text { s.t. }\left[b_{j}=1\right]_{j=1}^{R}\right\}
$$

where $b_{j}=1$ if the S-R link corresponding to the $j$ th relay is not in outage and $b_{j}=0$ if this link is in outage. Mathematically, this can be expressed as

$$
b_{j}= \begin{cases}1, & \text { if } \alpha_{\mathrm{s}, j} \geq \frac{\gamma_{\mathrm{th}}+\epsilon}{f_{j}}, \\ 0, & \text { otherwise, }\end{cases}
$$

where $\epsilon \geq 0$ is the margin value that needs to be optimized for achieving the best performance (cf. Section IV.A). The relay selection rule at the destination node is then given by

$$
q=\arg \max _{j \in \mathcal{A}}\left\{g_{j} \alpha_{j, \mathrm{~d}}\right\} .
$$

It can be seen from (7) that the relay with the best R-D channel may be associated with the worst S-R channel, i.e., the channel with SNR very close to $\gamma_{\mathrm{th}}$. Due to this reason and $\gamma_{j} \leq \min \left(f_{j} \alpha_{\mathrm{s}, j}, g_{j} \alpha_{j, \mathrm{~d}}\right)$, i.e., $\gamma_{j}$ is limited by the smaller value between $f_{j} \alpha_{\mathrm{s}, j}$ and $g_{j} \alpha_{j, \mathrm{~d}}$, it is clear that selecting the relay with the best R-D channel may not be sufficient for minimizing the outage in end-to-end transmission from the source to the destination. As such, $\epsilon$ is included in (6) for providing better outage performance. The rule (7) also suggests that, in contrast to the F-CSI method, the relays do not require to send the instantaneous CSI of the S-R channels to the destination for selecting the relay. On the other hand, in contrast to PRS, effort is made to form a set of candidate relays and estimate the CSI of the corresponding R-D channels. It is also important to note that according to the above protocol, the resulting end-to-end transmission from the source to the destination can be subject to outage even if both S-R and R-D links corresponding to the selected relay are individually not in 
outage, i.e., $f_{q} \alpha_{\mathrm{s}, q} \geq \gamma_{\mathrm{th}}+\epsilon$, and $g_{q} \alpha_{q, \mathrm{~d}} \geq \gamma_{\mathrm{th}}$, respectively. In this case, $\gamma_{q}$ in (4) can still be smaller than $\gamma_{\mathrm{th}}$. For example, if $g_{q} \alpha_{q, \mathrm{~d}}=\gamma_{\mathrm{th}}$ and $f_{q} \alpha_{\mathrm{s}, q}=\gamma_{\mathrm{th}}+\epsilon$, then (4) reduces to

$$
\gamma_{q}=\gamma_{\mathrm{th}} \frac{\gamma_{\mathrm{th}}+\epsilon}{2 \gamma_{\mathrm{th}}+\epsilon+1} \leq \gamma_{\mathrm{th}}
$$

where the equality holds only for $\gamma_{\mathrm{th}}=0$, which implies that the outage occurs in the end-to-end transmission from the source to the destination. Furthermore, with an aim to simply the analysis to be presented in the succeeding sections, we define the sets $\overline{\mathcal{A}}$ and $\mathcal{E}_{R}$ as

$$
\begin{aligned}
\overline{\mathcal{A}} & =\left\{j, \text { s.t. }\left[\alpha_{j, \mathrm{~d}} \geq \frac{\gamma_{\mathrm{th}}}{g_{j}}, b_{j}=1\right]_{j=1}^{R}\right\} \\
\mathcal{E}_{R} & =\left\{j, \text { s.t. }\left[\left(\alpha_{j, \mathrm{~d}}<\frac{\gamma_{\mathrm{th}}}{g_{j}}\right) \cup\left(b_{j}=0\right)\right]_{j=1}^{R}\right\} .
\end{aligned}
$$

It is clear that the end-to-end transmissions through the relays of set $\mathcal{E}_{R}$ are certain to be in outage. Finally, after the destination determines the selected relay and the former broadcasts a vector of binary digits representing the index of the selected relay 5 , it sends CSI of the corresponding S-R channel to the destination for decoding the source signal. As such, the training phase ends and the data transmission phase follows. The conventional two-phase single-relay transmission is then employed for data transmission using the selected relay.

\section{B. Qualitative Assessment of Overhead and Complexity}

In this subsection, we qualitatively compare the training and communication overhead requirement, and the computational complexity of $\mathrm{CE}$ of the proposed method with the PRS and F-CSI methods, assuming that all three methods employ training-based CE [10], [11] .

In a PRS scheme [12], the relay that gives the maximum first-hop SNR is selected, i.e., the selected relay is $q=\arg \max _{j}\left\{f_{j} \alpha_{\mathrm{s}, j}\right\}$. This means that if the selection is performed at the source node, it requires knowledge of the amplitudes of all S-R channels. The S-R channels as seen by the single-antenna source node form a multiple-input single-output (MISO) channel from the relays to the source, which can be estimated at the source without using feedback information. Towards this end, we consider that each relay broadcasts a vector of training signal of length $N$ with the time duration $T_{m}$. Depending on tradeoff between training overhead and the complexity of $\mathrm{CE}$ (this will be clear subsequently), following two schemes are considered for the PRS method; $M_{1}$ ) the source estimates the S-R MISO channel and $M_{2}$ ) the source and destination estimate the S-R and R-D MISO channels, respectively. In both cases, we consider that the MISO channels are estimated with a linear CE technique that employs the optimal training signal design (with the assumption that $N \geq R$ ) proposed in [24]. Thus, the training overhead of the PRS method (both

\footnotetext{
${ }^{5}$ Similar overhead is also required in the PRS (where source broadcasts the index) and F-CSI methods.

${ }^{6}$ For making a fair comparison, we let all methods to perform at their optimum levels, and thus, the S-R and R-D channels are separately estimated. The approach of estimating the overall S-R-D channel [10], [11] for applications that do not involve relay selection, is not readily applicable for our purpose.
}

$M_{1}$ and $M_{2}$ ) for selecting the relay at the source is $T_{m}$. The source broadcasts the index of the selected relay, which requires $\log _{2}(R)$ bits. In order to confirm that the destination has all CSI required for decoding the received signal, the source forwards the quantized estimate of the S-R channel (both amplitude and phase) corresponding to the selected relay to the destination via the selected relay. Let $2\left(T_{\mathrm{fa}}+T_{\mathrm{fp}}\right)$, where $T_{\mathrm{fa}}$ and $T_{\mathrm{fp}}$ denote the time consumed by the quantized amplitude and phase estimates respectively, denote the overhead of this step. In case of $M_{1}$, the selected relay then broadcasts a training signal of duration $T_{\mathrm{s}}$ so that the destination estimates the corresponding R-D SISO channel. However, in $M_{2}$, it is clear that this step is not required as the destination has already estimated the R-D MISO channel using the training signals broadcast by the relays. Consequently, the respective training overheads of $M_{1}$ and $M_{2}$ are $T_{m}+T_{\mathrm{s}}+2\left(T_{\mathrm{fa}}+T_{\mathrm{fp}}\right)$ and $T_{m}+2\left(T_{\mathrm{fa}}+T_{\mathrm{fp}}\right)$. The communication overhead of $M_{1}$ is $\log _{2}(R)$ bits, whereas that of $M_{2}$ is $2 \log _{2}(R)$ bits. The additional communication overhead of $\log _{2}(R)$ bits for $M_{2}$ is due to the fact that the destination also needs to know the index of the selected relay for distinguishing the selected R-D SISO channel from the estimated R-D MISO channel. In terms of computational complexity of $\mathrm{CE}, M_{1}$ requires to estimate an $R \times 1 \mathrm{MISO}$ channel and a SISO channel, whereas $M_{2}$ requires to estimate two $R \times 1$ MISO channels.

In the OR with F-CSI, the selected $q$ th relay is $q=$ $\arg \max _{j}\left\{\frac{f_{j} \alpha_{\mathrm{s}, j} g_{j} \alpha_{j, \mathrm{~d}}}{f_{j} \alpha_{\mathrm{s}, j}+g_{j} \alpha_{j, \mathrm{~d}}+1}\right\}$, which requires complete CSI at the destination [8], [9]. This means that the relay nodes need to first estimate the S-R SISO channels, which is done by using the training signal (of duration $T_{\mathrm{S}}$ ) broadcast by the source. The relays then send the quantized amplitude estimates of the S-R channels to the destination, which involves an overhead of $R T_{\mathrm{fa}}$. We consider that each relay broadcasts a training signal of length $N$ and the destination estimates the R-D MISO channel using the design approach of [24], which has an overhead of $T_{m}$. Thus, the total overhead for relay selection is $T_{m}+T_{\mathrm{s}}+R T_{\mathrm{fa}}$. The destination broadcasts the index of the best relay, which requires $\log _{2}(R)$ bits. Finally, the best relay sends the quantized phase estimate of the corresponding S-R channel to the destination (required for signal decoding). Considering that this step has an overhead of $T_{\mathrm{fp}}$, the total training overhead of the F-CSI method is $T_{m}+T_{\mathrm{s}}+R T_{\mathrm{fa}}+T_{\mathrm{fp}}$, whereas the communication overhead is $\log _{2}(R)$. This method requires to estimate the R-D MISO channel and $R$ SISO channels.

As in F-CSI method, the relays in the proposed method estimate the S-R SISO channels with a training overhead of $T_{\mathrm{s}}$. All non-candidate relays remain idle, whereas each candidate relay broadcasts its optimum training signal of duration $T_{m}$, which is designed on the basis of linear CE method for the R-D MISO channel [24]7. The training overhead of this step is $T_{m}$, meaning that the total overhead of relay selection is $T_{m}+T_{\mathrm{s}}$.

\footnotetext{
${ }^{7}$ We should note that, although the destination requires to estimate the R-D MISO channel corresponding to only the candidate relays, this approach of designing the optimal training signals for the complete R-D MISO channel is employed to enable the destination broadcast the index (w.r.t. the set of all relays) of the selected relay without additional communication overhead, for example, without informing the destination the state (either candidate or non-candidate) of each relay.
} 
TABLE I

QuALITATIVE COMPARISON OF OVERHEAD AND COMPLEXITY $\left(b_{\mathrm{v}} \leq 1\right)$.

\begin{tabular}{|c|c|c|c|}
\hline Method & Training Overhead & Number of CEs & Communication Overhead \\
\hline \multirow{2}{*}{ PRS } & $M_{1}: T_{\mathrm{m}}+T_{\mathrm{s}}+2\left(T_{\mathrm{fa}}+T_{\mathrm{fp}}\right)$ & $M_{1}: 1-R \times 1 \mathrm{MISO}, 1 \mathrm{SISO}$ & $M_{1}: \log _{2}(R)$ bits \\
& $M_{2}: T_{\mathrm{m}}+2\left(T_{\mathrm{fa}}+T_{\mathrm{fp}}\right)$ & $M_{2}: 2-R \times 1 \mathrm{MISO}$ & $M_{2}: 2 \log _{2}(R)$ bits \\
\hline Proposed & $T_{\mathrm{m}}+T_{\mathrm{s}}+b_{\mathrm{v}}\left(T_{\mathrm{fa}}+T_{\mathrm{fp}}\right)$ & $1-R \times 1 \mathrm{MISO}, R-$ SISO & $\log _{2}(R)$ bits \\
\hline F-CSI & $T_{\mathrm{m}}+T_{\mathrm{s}}+R T_{\mathrm{fa}}+T_{\mathrm{fp}}$ & $1-R \times 1 \mathrm{MISO}, R-$ SISO & $\log _{2}(R)$ bits \\
\hline
\end{tabular}

The destination selects the relay with the best R-D channel. Due to the property of the optimal training signals (see (9) and (31) in [24]), the selected relay simply turns to be the candidate relay with the best R-D channel. The destination then broadcasts the index of the selected relay (w.r.t. to the set of all relays) using $\log _{2}(R)$ bits. Notice that, whenever there is a candidate relay, the relay can be selected. The selected relay then forwards the quantized channel estimate (both amplitude and phase) of the corresponding S-R link to the destination (for decoding signal), which requires an overhead of $T_{\mathrm{fa}}+T_{\mathrm{fp}}$. However, if no relay can be selected, i.e., when $\mathcal{A}$ is empty, this step and the associated overhead do not come into effect. Thus, the average overhead of the step after the relay selection is $b_{\mathrm{v}}\left(T_{\mathrm{fa}}+T_{\mathrm{fp}}\right)$, where $b_{\mathrm{v}}=\operatorname{Pr}\{\mathcal{A} \neq \phi\}=\prod_{j=1}^{R} \operatorname{Pr}\left\{b_{j}=1\right\}$, and $\phi$ denotes a null set. Thus, the total average training overhead of the proposed scheme is $T_{m}+T_{\mathrm{s}}+b_{\mathrm{v}}\left(T_{\mathrm{fa}}+T_{\mathrm{fp}}\right)$, whereas the communication overhead is $\log _{2}(R)$ bits. The proposed method also estimates the $R \times 1$ R-D MISO channel and $R$ SISO channels.

The qualitative measure of the training overhead, communication overhead (in bits) and computational complexity of CE for the three relay selection methods is summarized in Table I. It is clear that the training overhead of the proposed scheme is always less than that of $M_{1}$ and does not exceed that of $M_{2}$ if $T_{\mathrm{s}} \leq\left(2-b_{\mathrm{v}}\right)\left(T_{\mathrm{fa}}+T_{\mathrm{fp}}\right)$. In the worst-case scenario, i.e., $b_{\mathrm{v}}=1$, the additional training overhead of the proposed scheme, when compared to $M_{2}$ of the PRS method, is independent of $R$ and only $T_{\mathrm{s}}-\left(T_{\mathrm{fa}}+T_{\mathrm{fp}}\right)$. Furthermore, even in the worst-case scenario, the proposed scheme's training overhead is less than that of the F-CSI method by $(R-1) T_{\mathrm{fa}}$, which is a significant improvement for larger values of $R$. The communication overhead (in bits) of the proposed method is the same as in $M_{1}$ of the PRS and F-CSI methods.

It is clear from Table I that the proposed scheme requires to estimate a larger number of SISO channels than the PRS scheme. However, the complexity of estimating each SISO channel is roughly equal to the complexity of finding an inner product between two vectors (see (9) of [21]). Nevertheless, this linear order of complexity and the complexity associated with the optimization of $\epsilon$, which requires low-cost one-dimensional line search (cf. Section IV.A), may be viewed as a price worth paying for significant performance gains over PRS.

Remark 1: Alternatively, it is possible to execute the relay selection at the source by selecting the candidate relay that gives the maximum S-R SNR. In this case, the candidate relays are the relays with their R-D links not in outage. Obviously, the destination and relays broadcast training signals, whereas the source and relays estimate their respective S-R MIMO and R-D SISO channels. The performance of this approach, in terms of the outage probability and diversity, will be the same as in the proposed method. However, the total training overhead turns to be larger than that of the proposed method. This is due to the fact that the required training for optimum decoding at the destination increases in the alternative method. More specifically, the source has to forward the estimate of the S-R channel, corresponding to the selected relay, to the destination via the selected relay. It also needs to forward the estimate of the corresponding R-D channel to the destination.

\section{Performance Analysis}

In this section, we analyze the performance of the proposed selection method assuming that the estimated channels approximate the actual channels with very high accuracy. Towards this end, we derive an exact expression for the outage probability at the destination, which is a widely used performance metric for fading channels. The outage probability for the proposed selection is defined as $P_{\mathrm{o}, R}=\operatorname{Pr}\left\{\frac{f_{q} \alpha_{\mathrm{s}, q} g_{q} \alpha_{q, \mathrm{~d}}}{f_{q} \alpha_{\mathrm{s}, q}+g_{q} \alpha_{q, \mathrm{~d}}+1} \leq \gamma_{\mathrm{th}}\right\}$ where $q$ is the index of the selected relay. It is clear that $P_{\mathrm{o}, R}$ depends on $q$, which in turn depends on the state of $\overline{\mathcal{A}}$. Let $\mathcal{P}$ denote the outage event at the destination. Using total probability law [25], $P_{\mathrm{o}, R}$ can be alternatively expressed as

$$
\begin{gathered}
P_{\mathrm{o}, R}=\sum_{l=1}^{2^{R}-1}\left[\operatorname{Pr}\left\{\mathcal{P} \mid \overline{\mathcal{A}}_{l}\right\} \operatorname{Pr}\left\{\overline{\mathcal{A}}_{l}\right\} \triangleq \operatorname{Pr}\left\{\mathcal{P}, \overline{\mathcal{A}}_{l}\right\}\right] \\
+\operatorname{Pr}\left\{\mathcal{E}_{R}\right\}
\end{gathered}
$$

where $2^{R}-1$ is the total number of possible states for $\overline{\mathcal{A}}$ and $\overline{\mathcal{A}}_{l}$ denotes the corresponding $l$ th state. Again with the total probability law, $\operatorname{Pr}\left\{\mathcal{P}, \overline{\mathcal{A}}_{l}\right\}$ can be expressed as

$$
\begin{aligned}
\operatorname{Pr}\left\{\mathcal{P}, \overline{\mathcal{A}}_{l}\right\}= & \sum_{k=1}^{L_{\overline{\mathcal{A}}_{l}}} \operatorname{Pr}\left\{\left[\mathcal{P}, \overline{\mathcal{A}}_{l}\right] \mid \alpha_{\overline{\mathcal{A}}_{l}(k), \mathrm{d}} \geq \max _{j \neq \overline{\mathcal{A}}_{l}(k), j \in \overline{\mathcal{A}}_{l}} \alpha_{j, \mathrm{~d}}\right\} \\
& \times \operatorname{Pr}\left\{\alpha_{\overline{\mathcal{A}}_{l(k), \mathrm{d}}} \geq \max _{j \neq \overline{\mathcal{A}}_{l}(k), j \in \overline{\mathcal{A}}_{l}} \alpha_{j, \mathrm{~d}}\right\}
\end{aligned}
$$

where $L_{\overline{\mathcal{A}}_{l}}$ is the cardinality of $\overline{\mathcal{A}}_{l}$ and $\overline{\mathcal{A}}_{l}(k)$ is the $k$ th element of $\overline{\mathcal{A}}_{l}$. It is clear from (11) and (12) that in order to obtain $P_{\mathrm{o}, R}$, $\operatorname{Pr}\left\{\mathcal{P}, \overline{\mathcal{A}}_{l}\right\}$ should be computed for all $l$. However, it is worthwhile to notice that the methodology for deriving $\operatorname{Pr}\left\{\mathcal{P}, \overline{\mathcal{A}}_{\bar{l}}\right\}$ and $\operatorname{Pr}\left\{\mathcal{P}, \overline{\mathcal{A}}_{\tilde{l}}\right\}$ remains same as long as $L_{\overline{\mathcal{A}}_{\bar{l}}}=L_{\overline{\mathcal{A}}_{\tilde{l}}}$. This means that when the channels in each hop are identically distributed, i.e., $f_{j}=f, g_{j}=g$, for all $j=1, \ldots, R$, it is sufficient to compute either $\operatorname{Pr}\left\{\mathcal{P}, \overline{\mathcal{A}}_{\bar{l}}\right\}$ or $\operatorname{Pr}\left\{\mathcal{P}, \overline{\mathcal{A}}_{\bar{l}}\right\}$. Due to these reasons, without loss of generality (w.l.o.g.) but for the sake of notational convenience and concise derivations, we assume that $f_{j}=f, g_{j}=g, \forall j$. Note that similar assumption is also made 
in previous studies with AF relays, e.g., in [12] and [13]. Moreover, the outage probability performance of the proposed selection for non-identical channels will be evaluated using simulations (cf. Section V.B). For better exposition and tractability of the analysis, we first derive expressions for $R \leq 3$ and then generalize the results to an arbitrary value of $R$.

\section{A. Outage Probability for $R=2,3$}

For $R=2$, the possible states of $\overline{\mathcal{A}}$ can be ordered as $\overline{\mathcal{A}}_{1}=$ $\{1,2\}, \overline{\mathcal{A}}_{2}=\{1\}$ and $\overline{\mathcal{A}}_{3}=\{2\}$. Similarly, for $R=3$, the possible states of $\overline{\mathcal{A}}$ can be ordered as $\overline{\mathcal{A}}_{1}=\{1,2,3\}, \overline{\mathcal{A}}_{2}=$ $\{1,2\}, \overline{\mathcal{A}}_{3}=\{1,3\}, \overline{\mathcal{A}}_{4}=\{2,3\}, \overline{\mathcal{A}}_{5}=\{1\}, \overline{\mathcal{A}}_{6}=\{2\}$ and $\overline{\mathcal{A}}_{7}=\{3\}$. Since we consider that $f_{j}=f, g_{j}=g, \forall j$, the same values of $\operatorname{Pr}\left\{\mathcal{P}, \overline{\mathcal{A}}_{l}\right\}$ are obtained for $l \in[2,3]$ when $R=2$. For $R=3$, the respective values of $\operatorname{Pr}\left\{\mathcal{P}, \overline{\mathcal{A}}_{l}\right\}$ for $l \in[5,6,7]$ and $l \in[2,3,4]$ are equal. Moreover, for both $R=2$ and $R=3$, each term of the summation in (12) is equal. Thus, using (11) and (12) and w.l.o.g., taking $\mathcal{P}=\left[\gamma_{1} \leq \gamma_{\mathrm{th}}\right]$, the outage probability for $R=2$ can be expressed as

$$
\begin{aligned}
P_{\mathrm{o}, 2}=2 \operatorname{Pr}\left\{\gamma_{1} \leq \gamma_{\text {th }} \mid \mathcal{B}_{2}\right\} \operatorname{Pr}\left\{\mathcal{B}_{2}\right\} & \\
& +2 \operatorname{Pr}\left\{\gamma_{1} \leq \gamma_{\text {th }} \mid \mathcal{B}_{1}\right\} \operatorname{Pr}\left\{\mathcal{B}_{1}\right\}+\operatorname{Pr}\left\{\mathcal{E}_{2}\right\}
\end{aligned}
$$

where

$$
\begin{aligned}
& \mathcal{B}_{1}=\left\{\alpha_{1, \mathrm{~d}} \geq \frac{\gamma_{\mathrm{th}}}{g}, b_{1}=1,\left[\alpha_{2, \mathrm{~d}}<\frac{\gamma_{\mathrm{th}}}{g} \cup b_{2}=0\right]\right\}, \\
& \mathcal{B}_{2}=\left\{\alpha_{1, \mathrm{~d}} \geq \alpha_{2, \mathrm{~d}},\left[\alpha_{j, \mathrm{~d}}\right]_{j=1}^{2} \geq \frac{\gamma_{\mathrm{th}}}{g},\left[b_{j}=1\right]_{j=1}^{2}\right\} .
\end{aligned}
$$

$\mathcal{B}_{1}$ and $\mathcal{B}_{2}$ are two disjoint sets. Similarly, for $R=3$, the outage probability can be expressed as

$$
\begin{gathered}
P_{\mathrm{o}, 3}=3 \operatorname{Pr}\left\{\gamma_{1} \leq \gamma_{\mathrm{th}} \mid \widetilde{\mathcal{B}}_{3}\right\} \operatorname{Pr}\left\{\widetilde{\mathcal{B}}_{3}\right\}+6 \operatorname{Pr}\left\{\gamma_{1} \leq \gamma_{\mathrm{th}} \mid \widetilde{\mathcal{B}}_{2}\right\} \\
\operatorname{Pr}\left\{\widetilde{\mathcal{B}}_{2}\right\}+3 \operatorname{Pr}\left\{\gamma_{1} \leq \gamma_{\mathrm{th}} \mid \widetilde{\mathcal{B}}_{1}\right\} \operatorname{Pr}\left\{\widetilde{\mathcal{B}}_{1}\right\}+\operatorname{Pr}\left\{\mathcal{E}_{3}\right\}
\end{gathered}
$$

where the sets $\widetilde{\mathcal{B}}_{1}, \widetilde{\mathcal{B}}_{2}$ and $\widetilde{\mathcal{B}}_{3}$ are disjoint, and are defined as

$$
\begin{gathered}
\widetilde{\mathcal{B}}_{1}=\left\{\alpha_{1, \mathrm{~d}} \geq \frac{\gamma_{\mathrm{th}}}{g}, b_{1}=1,\left[\alpha_{2, \mathrm{~d}}<\frac{\gamma_{\mathrm{th}}}{g} \cup b_{2}=0\right],\right. \\
\left.\left[\alpha_{3, \mathrm{~d}}<\frac{\gamma_{\mathrm{th}}}{g} \cup b_{3}=0\right]\right\}, \\
\widetilde{\mathcal{B}}_{2}=\left\{\alpha_{1, \mathrm{~d}} \geq \alpha_{2, \mathrm{~d}},\left[\alpha_{j, \mathrm{~d}}\right]_{j=1}^{2} \geq \frac{\gamma_{\mathrm{th}}}{g},\left[b_{j}=1\right]_{j=1}^{2},\right. \\
\left.\left[\alpha_{3, \mathrm{~d}}<\frac{\gamma_{\mathrm{th}}}{g} \cup b_{3}=0\right]\right\}, \\
\widetilde{\mathcal{B}}_{3}=\left\{\alpha_{1, \mathrm{~d}} \geq \max \left(\alpha_{2, \mathrm{~d}}, \alpha_{3, \mathrm{~d}}\right),\left[\alpha_{j, \mathrm{~d}}\right]_{j=1}^{3} \geq \frac{\gamma_{\mathrm{th}}}{g},\right. \\
\left.\left[b_{j}=1\right]_{j=1}^{3}\right\} .
\end{gathered}
$$

The outage probabilities of (15) and (13) consist of (conditional) joint probability terms, each having statistically dependent random events with two or more continuous random variables. Thus, derivation of exact expressions requires solving multidimensional integrals which need rigorous analysis. As one of the key contributions of this section, we now proceed to derive the exact expressions of $P_{\mathrm{o}, 3}$ for $\epsilon \geq 0$. The expressions for $P_{\mathrm{o}, 2}$ follow easily from the expressions of $P_{\mathrm{o}, 3}$. To this end, we have the following proposition.

Proposition 1: The outage probability for $R=3$ and $\epsilon \geq 0$ can be expressed as

$$
P_{\mathrm{o}, 3}=3 \mathrm{e}^{-2 d_{2}} r_{3}+6 \mathrm{e}^{-d_{2}} r_{2} r_{0}+3 r_{1} r_{0}^{2}+r_{0}^{3}
$$

where

$$
\begin{aligned}
& r_{3}=\mathrm{e}^{-\gamma_{\mathrm{th}}\left(\frac{3}{g}+\frac{1}{f}\right)}\left\{\frac{\mathrm{e}^{-\frac{\epsilon}{f}}}{3}+s_{2}-s_{1}-\frac{1}{3} s_{3}\right\}, \\
& r_{2}=\mathrm{e}^{-\gamma_{\mathrm{th}}\left(\frac{2}{g}+\frac{1}{f}\right)}\left\{\frac{1}{2} \mathrm{e}^{-\frac{\epsilon}{f}}+\frac{1}{2} s_{2}-s_{1}\right\}, \\
& r_{1}=\mathrm{e}^{-\gamma_{\mathrm{th}}\left(\frac{1}{g}+\frac{1}{f}\right)}\left(\mathrm{e}^{-\frac{\epsilon}{f}}-s_{1}\right), d_{2}=\frac{\epsilon+\gamma_{\mathrm{th}}}{f} \\
& r_{0}=\left[1-\mathrm{e}^{-\frac{\epsilon}{f}} \mathrm{e}^{-\gamma_{\mathrm{th}}\left(\frac{1}{g}+\frac{1}{f}\right)}\right] .
\end{aligned}
$$

Furthermore, $\left\{s_{m}\right\}_{m=1}^{3}$ in (18) is expressed as

$$
\begin{aligned}
s_{m}^{(\epsilon=0)}= & 2 \sqrt{\frac{m \gamma_{\mathrm{th}}\left(\gamma_{\mathrm{th}}+1\right)}{g f}} K_{1}\left(2 \sqrt{\frac{m \gamma_{\mathrm{th}}\left(\gamma_{\mathrm{th}}+1\right)}{g f}}\right) \\
s_{m}^{(\epsilon>0)}= & \mathrm{e}^{-\frac{\epsilon}{f}}+\sum_{k=1}^{\infty}\left(-m \gamma_{\mathrm{th}}\left(\gamma_{\mathrm{th}}+1\right)\right)^{k} \frac{g^{-k}}{k !}(\epsilon f)^{-\frac{k}{2}} \mathrm{e}^{-\frac{\epsilon}{2 f}} \\
& \times W_{-\frac{k}{2}, \frac{1-k}{2}}\left(\frac{\epsilon}{f}\right)
\end{aligned}
$$

where $K_{1}($.$) is the first order modified Bessel function of the$ second type and $W_{\lambda, \mu}($.$) is the Whittaker function [26] with the$ parameters $\lambda$ and $\mu$.

Proof: Please refer to Appendix A.

From Proposition 1, the outage probability expression for $R=2$ readily follows, which is given by

$$
P_{\mathrm{o}, 2}=2 \mathrm{e}^{-d_{2}} r_{2}+2 r_{1} r_{0}+r_{0}^{2} .
$$

The expressions (17) and (21) (both for $\epsilon>0$ ) can also be computed efficiently, since the series of Whittaker function converges after a few terms, especially for medium to high values of both $g$ and $f$.

\section{B. Generalizing Outage Probability to an Arbitrary $R$}

Based upon expressions for $P_{\mathrm{o}, 2}$ and $P_{\mathrm{o}, 3}$, we derive expressions for $P_{\mathrm{o}, R}$ where $R>3$. It is clear from (17) and (21) that $r_{0}, r_{1}$ and $r_{2}$ of $P_{\mathrm{o} .2}$ also appear in the last three terms of $P_{\mathrm{o} .3}$. This means that when $P_{\mathrm{o}, 2}$ is obtained, it only requires to obtain $r_{3}$ for getting expression for $P_{\mathrm{o}, 3}$. Recall that the method for obtaining $r_{3}$ is given in Appendix A. Based on this information ((17)-(18) and (21)) and all possible states of $\overline{\mathcal{A}}$, general expression for the outage probability can be obtained from mathematical induction as

$$
\begin{aligned}
P_{\mathrm{o}, 4}= & 4 \mathrm{e}^{-3 d_{2}} r_{4}+12 \mathrm{e}^{-2 d_{2}} r_{3} r_{0}+12 \mathrm{e}^{-d_{2}} r_{2} r_{0}^{2}+4 r_{1} r_{0}^{3}+r_{0}^{4}, \\
P_{\mathrm{o}, 5}= & 5 \mathrm{e}^{-4 d_{2}} r_{5}+20 \mathrm{e}^{-3 d_{2}} r_{4} r_{0}+30 \mathrm{e}^{-2 d_{2}} r_{3} r_{0}^{2} \\
& +20 \mathrm{e}^{-d_{2}} r_{2} r_{0}^{3}+5 r_{1} r_{0}^{4}+r_{0}^{5}
\end{aligned}
$$




$$
P_{\mathrm{o}, R}=r_{0}^{R}+\sum_{l=1}^{R} \frac{R !}{(R-l) !(l-1) !} \mathrm{e}^{-(l-1) d_{2}} r_{0}^{R-l} r_{l},
$$

which means that we have to obtain the expression for $r_{l}$, where $l=1, \ldots, R$. Notice that generalizing the expression of $r_{3}$ (see (33) of Appendix A), $r_{l}$ can be expressed as

$$
\begin{array}{r}
r_{l}=\operatorname{Pr}\left\{\gamma_{1} \leq \gamma_{\mathrm{th}}, \alpha_{1, \mathrm{~d}} \geq \max \left(\alpha_{2, \mathrm{~d}}, \ldots, \alpha_{l, \mathrm{~d}}\right), b_{1}=1,\right. \\
\left.\left\{\alpha_{j, \mathrm{~d}}\right\}_{j=1}^{l} \geq \frac{\gamma_{\mathrm{th}}}{g}\right\} .
\end{array}
$$

Substituting $\gamma_{1}$ into (23) and using similar steps as (34)-(35) of Appendix A, (23) can be written as the following multidimensional integral

$$
\begin{aligned}
r_{l}= & \int_{y=\frac{\gamma_{\mathrm{th}}+\epsilon}{f}}^{\infty} \int_{u_{l}=\frac{\gamma_{\mathrm{th}}}{g}}^{\bar{u}} \cdots \int_{u_{3}=\frac{\gamma_{\mathrm{th}}}{g}}^{\bar{u}} \int_{u_{2}=\frac{\gamma_{\mathrm{th}}}{g}}^{\bar{u}} \operatorname{Pr}\left\{\alpha_{1, \mathrm{~d}} \leq\right. \\
& \left.\frac{\gamma_{\mathrm{th}}(f y+1)}{g f y-g \gamma_{\mathrm{th}}}, \alpha_{1, \mathrm{~d}} \geq u_{2}, \ldots, \alpha_{l, \mathrm{~d}} \geq u_{l}\right\} \\
& \times f_{\alpha_{2, \mathrm{~d}}}\left(u_{2}\right) d u_{2} \cdots f_{\alpha_{l, \mathrm{~d}}}\left(u_{l}\right) d u_{l} f_{\alpha_{\mathrm{s}, 1}}(y) d y
\end{aligned}
$$

where $\bar{u}=\frac{\gamma_{\mathrm{th}}(f y+1)}{g f y-g \gamma_{\mathrm{th}}}$, and $\left\{f_{\alpha_{l, \mathrm{~d}}}\right\}$ and $f_{\alpha_{\mathrm{s}, 1}}$ are respectively, the PDFs of $\left\{\alpha_{l, \mathrm{~d}}\right\}$ and $\alpha_{\mathrm{s}, 1}$. Then, the main result is stated in the following proposition.

Proposition 2: The expression for $r_{l}$, is given by

$$
r_{l}=(l-1) ! \mathrm{e}^{-\gamma_{\mathrm{th}}\left(\frac{l}{g}+\frac{1}{f}\right)} \sum_{m=0}^{l} \frac{(-1)^{m}}{m !(l-m) !} s_{m}
$$

where $s_{0}^{(\epsilon>0)}=\mathrm{e}^{-\frac{\epsilon}{f}}$ and $s_{0}^{(\epsilon=0)}=1$.

Proof: Please refer to Appendix B.

With this expression for $r_{l}$, the exact outage probability (22) is obtained for $\epsilon \geq 0$. The following remark is in order.

Remark 2: By writing (22) in terms of a general variable $x$, where $x \triangleq \gamma_{\text {th }}$ and differentiating the resulting expression w.r.t. $x$, the probability density function (PDF) of $\gamma_{q}$ can be determined. This PDF may be used for analyzing other important performance metrics in fading channels. Due to space constraints, we skip the related details in this paper.

\section{Asymptotic ANALYSIS}

In this section, we present asymptotic analysis of the outage probability for revealing the diversity gain ${ }^{8}$ of the proposed relay selection method. We first obtain asymptotic results for $\epsilon=0$ and show that $\epsilon=0$ does not achieve full diversity when $R \geq 3$. In this case, the key idea is to employ a general asymptotic expression for the function $K_{1}(x)$ when $x \rightarrow 0$, since the standard expression (e.g., used in [20]) is not applicable for getting the diversity order. We then determine asymptotic expression with $\epsilon>0$ and show that full diversity is achieved for arbitrary $R$. In this case, the asymptotic expression of $W_{\lambda, \mu}(z)$

\footnotetext{
${ }^{8}$ Diversity gain is defined as the slope of the outage probability curve in logscale at the high SNR regimes, i.e., $d_{\text {asymptotic }}=-\lim _{\rho \rightarrow \infty} \frac{\log P_{0, R}}{\log \rho}$, where $\rho$ is a common average SNR and defined as $\rho \triangleq g=f[3]$.
}

$(z \rightarrow 0)$ is utilized. Towards this end, the first main result is presented below.

Proposition 3: For $R=2$ and $\epsilon=0$, the asymptotic expression for $P_{\mathrm{o}, 2}$ is given by

$$
\begin{aligned}
P_{\mathrm{o}, 2}^{(\epsilon=0)} \approx 2 \gamma_{\mathrm{th}}\left(\gamma_{\mathrm{th}}+1\right) \log (2)(g f)^{-1} & \\
& +\gamma_{\mathrm{th}}^{2}\left(\frac{1}{g}+\frac{1}{f}\right)^{2}+\mathcal{O}\left((g f)^{-1}\right),
\end{aligned}
$$

which means that the proposed scheme achieves full diversity order of 2.

Proof: The asymptotic expression of $P_{\mathrm{o}, 2}$ is derived in Appendix C. W.1.o.g, we assume that $g=\bar{\gamma}$ and $f=\eta \bar{\gamma}$, where $\eta>0$. Then, it is clear from (26) that the outage probability for large values of $\bar{\gamma}$ is dominated by the lowest order term in $\frac{1}{\bar{\gamma}}$, which in this case is $\bar{\gamma}^{-2}$. This means that the diversity order of 2 is achieved for $R=2$ with $\epsilon=0$.

Proposition 4: For $R=3$ and $\epsilon=0$, the asymptotic expression for $P_{\mathrm{o}, 3}$ is given by

$$
\begin{aligned}
P_{\mathrm{o}, 3}^{(\epsilon=0)} \approx 3 \gamma_{\mathrm{th}}\left(\gamma_{\mathrm{th}}+\right. & 1)(g f)^{-1}(2 \log (2)-\log (3)) \\
& +\mathcal{O}\left((g f)^{-1}\right)
\end{aligned}
$$

which means that with $\epsilon=0$, only the diversity order of 2 is achieved for $R=3$.

Proof: Please refer to Appendix D.

From propositions 2 and 3 , we find that $\epsilon=0$ gives full diversity gain for $R=2$, but not for $R=3$. The reason is that $\epsilon=0$ is obviously not the best choice for optimal performance. This is due to the fact that the selection of the candidate relay with the maximum R-D SNR can easily result into the relay having the worst S-R SNR which can be very close to $\gamma_{\mathrm{th}}$. Consequently, outage can readily occur in end-to-end transmission from the source to the destination. The probability of this situation, however, can be avoided or significantly reduced, by properly selecting $\epsilon$. For better exposition of the aforementioned discussions, let us consider an example with $R=3, g=f=16 \mathrm{~dB}$, $\gamma_{\mathrm{th}}=3 \mathrm{~dB}$ (i.e., $\frac{\gamma_{\mathrm{th}}}{f}=0.0501$ ), and the channel realization yielding

$$
\begin{aligned}
& {\left[\alpha_{1, \mathrm{~d}}=0.9136, \alpha_{2, \mathrm{~d}}=0.1524, \alpha_{3, \mathrm{~d}}=0.4015\right],} \\
& {\left[\alpha_{\mathrm{s}, 1}=0.0524, \alpha_{\mathrm{s}, 2}=1.9847, \alpha_{\mathrm{s}, 3}=1.7240\right] .}
\end{aligned}
$$

In this case, all three relays are candidate relays. The selection method chooses the first relay, since this is the one that gives the maximum second-hop SNR. This selection results into the end-to-end SNR value of $\gamma_{1}=2.8427 \mathrm{~dB}$ leading to an outage. However, if the selection method had chosen either the second or third relay, the outage could have been avoided, since the second and third relays yield end-to-end SNR values of 7.4584 $\mathrm{dB}$ and $11.0765 \mathrm{~dB}$, respectively. By taking $\epsilon>0$, and in this particular case, $\epsilon=0.1 \gamma_{\mathrm{th}}$, the outage can then be avoided, since this value of $\epsilon$ rules out the selection of the first relay. With these discussions, we are now in a position to show analytically that the full diversity order can be achieved for $R \geq 3$ with $\epsilon>0$. We will also propose a method to optimize $\epsilon$, which 
further improves the performance. In this regard, the main result is presented in the following proposition:

Proposition 5: In the asymptotic region, i.e., when $g, f \rightarrow$ $\infty, r_{l} \approx r_{l}^{\mathrm{a}}$, where $r_{l}^{\mathrm{a}}$ can be expressed as

$$
r_{l}^{\mathrm{a}}= \begin{cases}-\tilde{\gamma}_{\mathrm{th}}(g f)^{-1}\left(\gamma_{\mathrm{c}}+\log \left(\frac{\epsilon}{f}\right)\right) & \text { if } l=1, \\ \frac{\tilde{\gamma}_{\mathrm{th}}^{2}}{l}(g f)^{-2} \log \left(\frac{\epsilon}{f}\right)-\frac{\tilde{\gamma}_{\mathrm{th}}^{3}}{l} \epsilon^{-2} g^{-3} f^{-1} & \text { if } l=2, \\ \frac{1}{l} \tilde{\gamma}_{\mathrm{th}}^{l} g^{-l} f^{-1} \epsilon^{1-l}(l-2) \text { if, } l>2, \ldots, R, & \end{cases}
$$

$\tilde{\gamma}_{\mathrm{th}}=\gamma_{\mathrm{th}}\left(\gamma_{\mathrm{th}}+1\right)$ and $\gamma_{\mathrm{c}}$ is Euler's constant [26].

Proof: The proof is based on the asymptotic expansion of Whittaker functions and given in Appendix E.

Notice that $\mathrm{e}^{-(l-1) d_{2}} \approx 1$ and $r_{0} \approx r_{0}^{\mathrm{a}}=\frac{\epsilon}{f}+\gamma_{\mathrm{th}}\left(\frac{1}{g}+\frac{1}{f}\right)$ when $f, g \rightarrow \infty$. Using these facts and substituting (28) into (22), the asymptotic expression of the outage probability for the case with $R$ relays is given by

$$
P_{\mathrm{o}, R}^{(\epsilon>0)} \approx\left(r_{0}^{\mathrm{a}}\right)^{R}+\sum_{l=1}^{R} \frac{R !}{(R-l) !(l-1) !}\left(r_{0}^{\mathrm{a}}\right)^{R-l} r_{l}^{\mathrm{a}}
$$

which can be expanded to the following form

$$
\begin{aligned}
& P_{\mathrm{o}, R}^{(\epsilon>0)} \approx\left[\frac{\epsilon}{f}+\gamma_{\mathrm{th}}\left(\frac{1}{g}+\frac{1}{f}\right)\right]^{R}-\left[\frac{\epsilon}{f}+\gamma_{\mathrm{th}}\left(\frac{1}{g}+\frac{1}{f}\right)\right]^{R-1} \\
& R \tilde{\gamma}_{\mathrm{th}}(g f)^{-1}\left[\gamma_{\mathrm{c}}+\log \left(\frac{\epsilon}{f}\right)\right]+\frac{R(R-1) \tilde{\gamma}_{\mathrm{th}}^{2} g^{-2} f^{-1}}{2} \\
& {\left[\frac{\epsilon}{f}+\gamma_{\mathrm{th}}\left(\frac{1}{g}+\frac{1}{f}\right)\right]^{R-2}\left[f^{-1} \log \left(\frac{\epsilon}{f}\right)-\tilde{\gamma}_{\mathrm{th}} \epsilon^{-2} g^{-1}\right]} \\
& +\sum_{l=3}^{R} \frac{(l-2) R !}{l !(R-l) !}\left[\frac{\epsilon}{f}+\gamma_{\mathrm{th}}\left(\frac{1}{g}+\frac{1}{f}\right)\right]^{R-l} \tilde{\gamma}_{\mathrm{th}}^{l} \epsilon^{1-l} g^{-l} f^{-1} .
\end{aligned}
$$

W.l.o.g., we assume that $g=\bar{\gamma}$ and $f=\eta \bar{\gamma}$, where $\eta>0$. Then, after analyzing (30) for a given $\epsilon>0$, we find that the dominant term, i.e., the term with the lowest exponent of $\frac{1}{\bar{\gamma}}$ is $\left[\frac{\epsilon}{\eta \bar{\gamma}}+\gamma_{\mathrm{th}}\left(\frac{1}{\bar{\gamma}}+\frac{1}{\eta \bar{\gamma}}\right)\right]^{R}$. This means that the diversity order of $R$ is achieved.

\section{A. Optimizing $\epsilon$}

The optimum value of $\epsilon$ is the one which minimizes the exact outage probability (22). However, the expression for the outage probability consists of a series of Whittaker functions. As such, it is difficult to obtain the optimum solution for $\epsilon$. Therefore, we employ the asymptotic expression (30) and obtain $\epsilon$ that is optimal under high SNR approximation. To this end, we solve the minimization problem $\min _{\epsilon>0} P_{\mathrm{o}, R}^{(\epsilon>0)}$ after further removing the terms with the higher orders of $g^{-1}$ and $f^{-1}$. The resulting objective functions can be better visualized for cases like $R=$ 2 and $R=3$, in which we have the following optimization problems

$$
\begin{gathered}
\min _{\epsilon}\left[\widetilde{P}_{\mathrm{o}, 3}^{(\epsilon>0)} \triangleq \tilde{\gamma}_{\mathrm{th}}^{3} g^{-3} f^{-1} \epsilon^{-2}-3 \tilde{\gamma}_{\mathrm{th}}(g f)^{-1}\left(\gamma_{\mathrm{c}}+\log \left(\frac{\epsilon}{f}\right)\right)\right. \\
\left.\left[\frac{\epsilon}{f}+\gamma_{\mathrm{th}}\left(g^{-1}+f^{-1}\right)\right]^{2}+\left[\frac{\epsilon}{f}+\gamma_{\mathrm{th}}\left(g^{-1}+f^{-1}\right)\right]^{3}\right],(31)
\end{gathered}
$$

$$
\begin{aligned}
& \min _{\epsilon}\left[\widetilde{P}_{\mathrm{o}, 2}^{(\epsilon>0)} \triangleq-2 \tilde{\gamma}_{\mathrm{th}}(g f)^{-1}\left(\gamma_{\mathrm{c}}+\log \left(\frac{\epsilon}{f}\right)\right)\right. \\
& \left.\left[\frac{\epsilon}{f}+\gamma_{\mathrm{th}}\left(\frac{1}{g}+\frac{1}{f}\right)\right]+\left[\frac{\epsilon}{f}+\gamma_{\mathrm{th}}\left(\frac{1}{g}+\frac{1}{f}\right)\right]^{2}\right]
\end{aligned}
$$

which are obtained from $P_{\mathrm{o}, 3}^{(\epsilon>0)}$ and $P_{\mathrm{o}, 2}^{(\epsilon>0)}$, respectively, after removing the higher order terms of $g^{-1}$ and $f^{-1}$. It is still difficult to solve (31) and (32) in closed-form. However, the minimization problems of (31) and (32) can be solved numerically, e.g., using one-dimensional search such as standard bisection method [27], which converges in $\log _{2}\left(\frac{u b-l b}{\tilde{\epsilon}}\right)$ iterations, where $\epsilon \in[l b, u b]$ and $\tilde{\epsilon}$ is the required convergence accuracy. Alternatively, we can evaluate $\widetilde{P}_{\mathrm{o}, 3}^{(\epsilon>0)}$ and $\widetilde{P}_{\mathrm{o}, 2}^{(\epsilon>0)}$ for a fine grid of $\epsilon>0$, and obtain the values of $\epsilon$ that give the minimum values of $\widetilde{P}_{\mathrm{o}, 3}^{(\epsilon>0)}$ and $\widetilde{P}_{\mathrm{o}, 2}^{(\epsilon>0)}$. Both of the aforementioned methods can be easily applied for $R>3$. We conclude this section with the following remarks:

Remark 3: We propose to solve the optimization problems (31) and (32) at the destination, which requires only the knowledge of $g$ and $f$, i.e., path gains of the S-R and R-D channels, transmit powers of the source and relays, and receiver noise powers. As long as the network deployment scenario does not change, it is clear that these parameters remain almost unchanged. This means that (31) and (32) are solved offline only for once and the optimal $\epsilon$ is made available to the relays like a system parameter.

Remark 4: With a motivation of achieving full diversity at a relatively low cost, the proposed local CSI based method considers single relay selection. On the other hand, for the systems having global CSI, it is known that multiple relay selection provides improved coding gains in addition to full diversity gain [9]. In this context, an extension of the proposed method to a multiple relay selection scheme remains as an open research topic and is left for future work.

Remark 5: The proposed method is developed for two-hop relay networks which are mainly suitable for cellular networks, especially in assisting the cell-edge users or users out of the coverage area of the source node (or the base station). In LTE standards [28], such relays are known as Type I relays. Moreover, a special case of the proposed selection, i.e., the case with $\epsilon=0$ can be viewed as a direct application of SC to AF networks. As a result, it is obvious that the proposed method is applicable for threshold-based DF networks [17]-[19]. Because maximum throughput in multihop relaying can be obtained with 2-3 hops [29] and can even decrease with more than 2-3 hops [30], the potential application of the proposed relay selection can also be extended to multihop networks. In particular, a closer analysis of the tradeoff between throughput and diversity [31] in multihop networks shows that it may be better to apply a $s u b$ optimal (in terms of outage performance or diversity gain of the multihop network) relay selection method having CSI of only two-hop channels than the optimal routing scheme [32] that requires large overhead for acquiring the global CSI of all multihops. The proposed scheme, in fact, can act as the aforementioned suboptimal selection scheme. Consequently, the proposed method can be executed in each two-hop segment of a multihop network. 


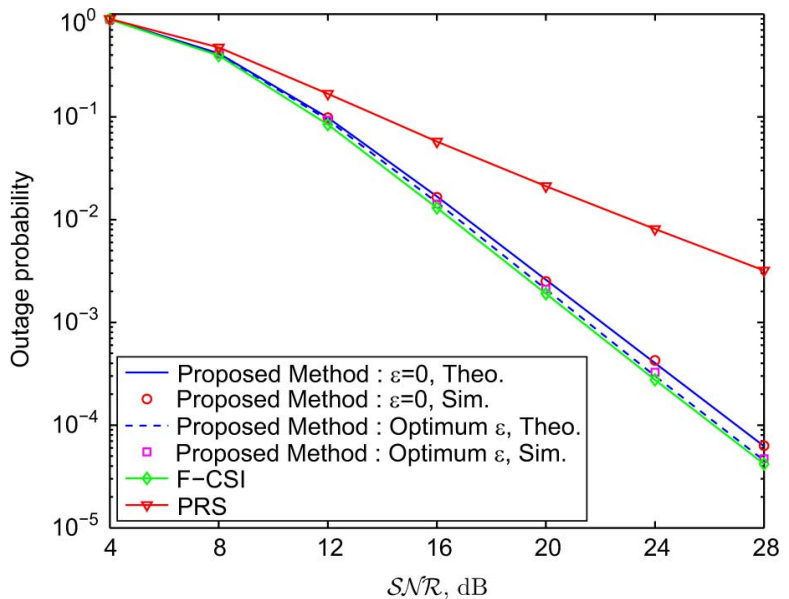

Fig. 1. Outage probability versus $\mathcal{S} \mathcal{N} \mathcal{R}$ for $R=2$.

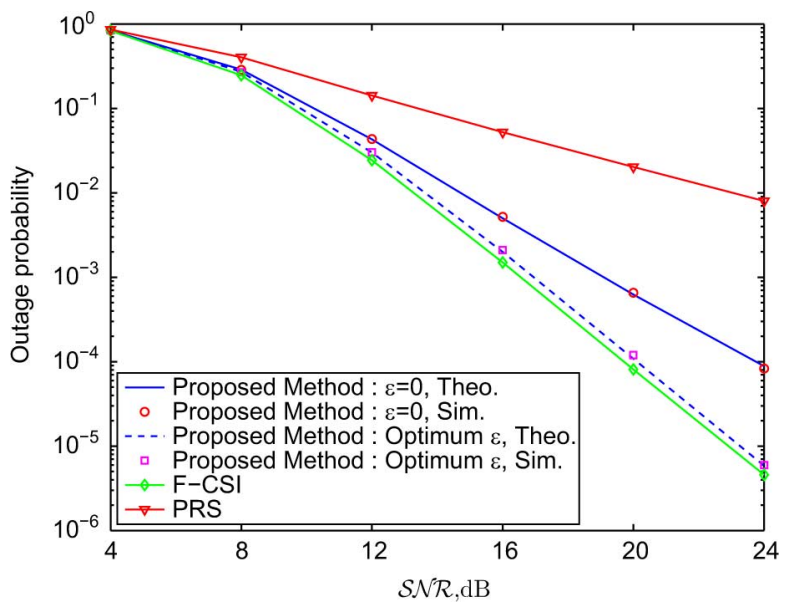

Fig. 2. Outage probability versus $\mathcal{S N} \mathcal{R}$ for $R=3$.

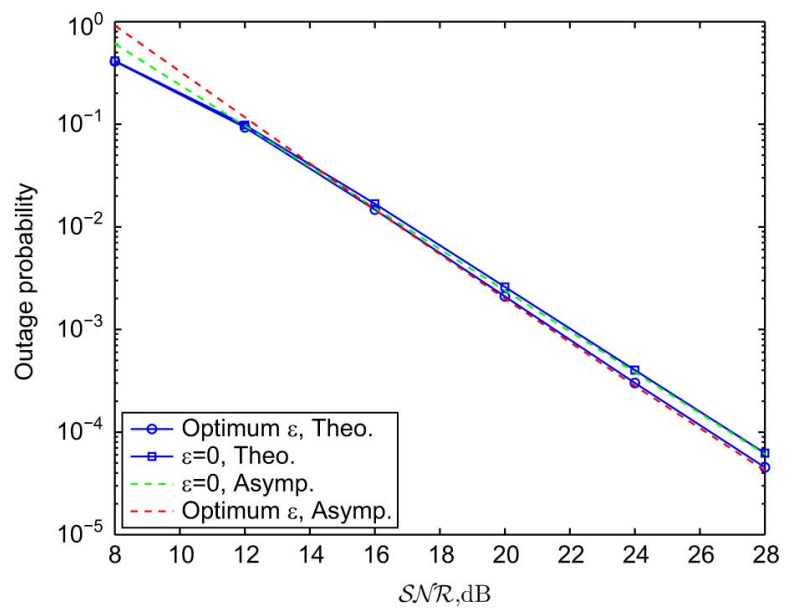

Fig. 3. Asymptotic and exact outage probabilities versus $\mathcal{S} \mathcal{N} \mathcal{R}$ for the proposed method $(R=2)$.

\section{NUMERICAL RESULTS}

In this section, we provide Monte Carlo simulations to assess the accuracy of the exact and asymptotic expressions for the outage probability. We also compare the performance of our

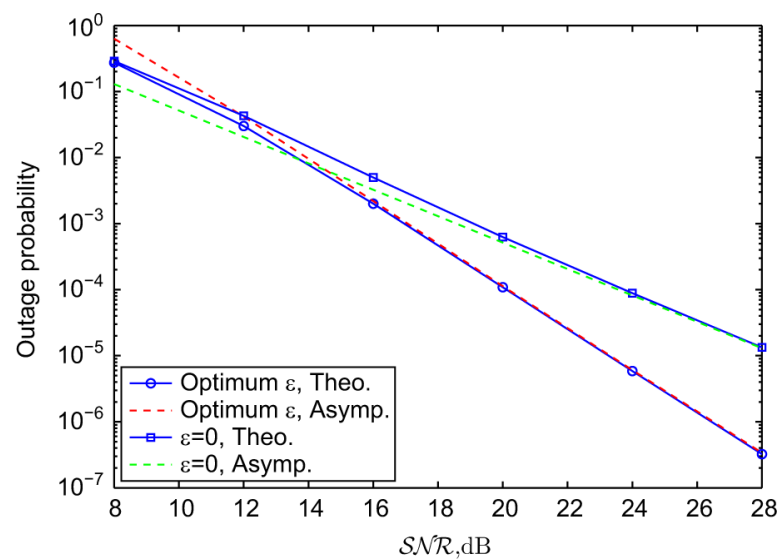

Fig. 4. Asymptotic and exact outage probabilities versus $\mathcal{S} \mathcal{N} \mathcal{R}$ for the proposed method $(R=3)$.

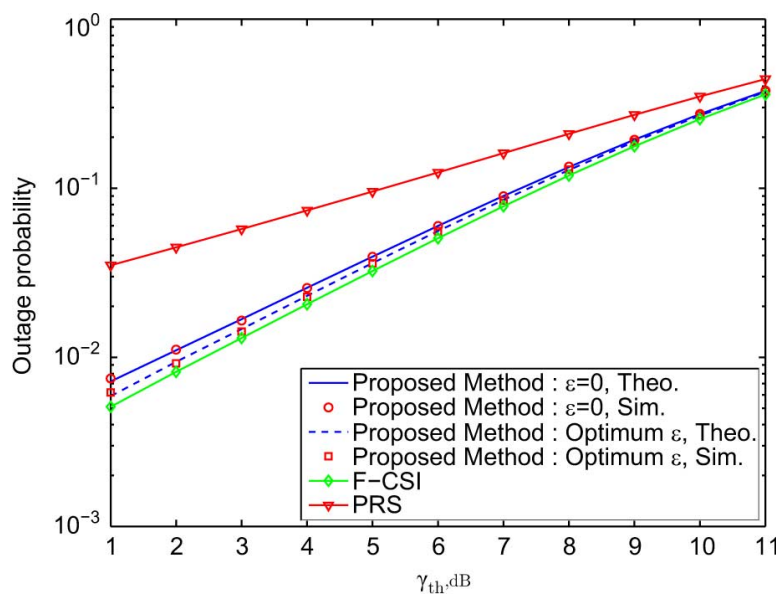

Fig. 5. Outage probability versus $\gamma_{\mathrm{th}}$ for $R=2$.

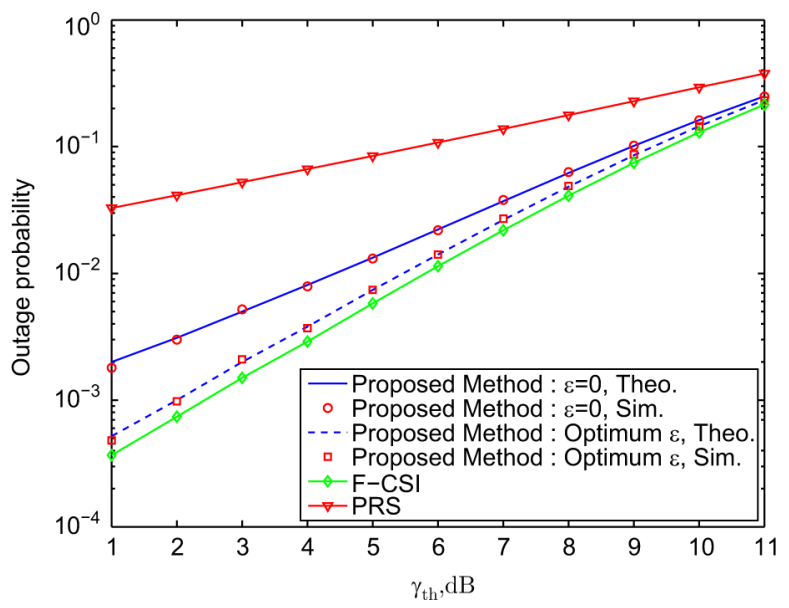

Fig. 6. Outage probability versus $\gamma_{\text {th }}$ for $R=3$.

proposed method with the PRS [12] and opportunistic relay selection based on F-CSI [13]. In all examples, we take $\gamma_{\mathrm{th}}=3$ $\mathrm{dB}$ (except in Figs. 5-6 and 9, where $\gamma_{\mathrm{th}}$ varies) and $\sigma_{j}^{2}=\sigma_{\mathrm{d}}^{2}=$ $\sigma_{n}^{2}$. All fading channels are taken to be ZMCSCG random variables with unit variance. 


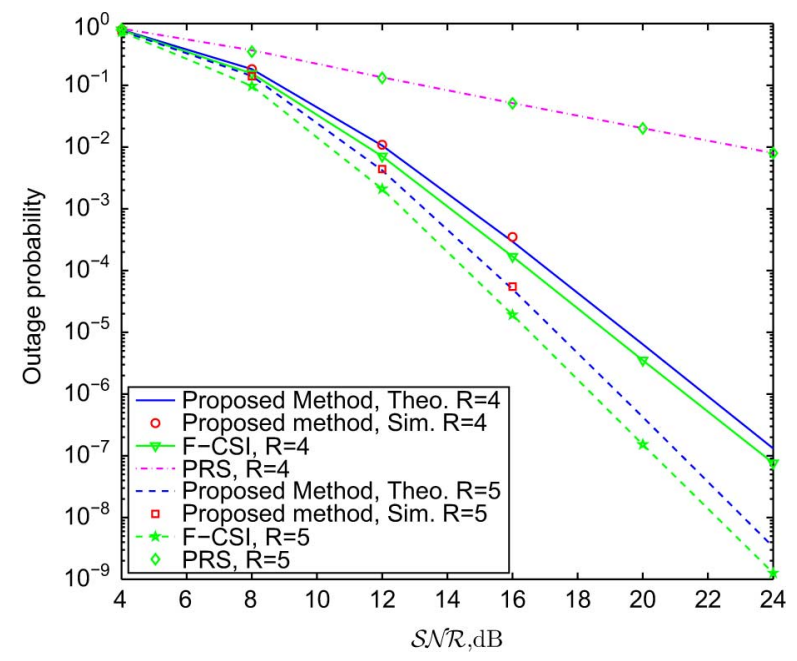

Fig. 7. Outage probability versus $\mathcal{S N} \mathcal{R}$ for $R=4,5$.

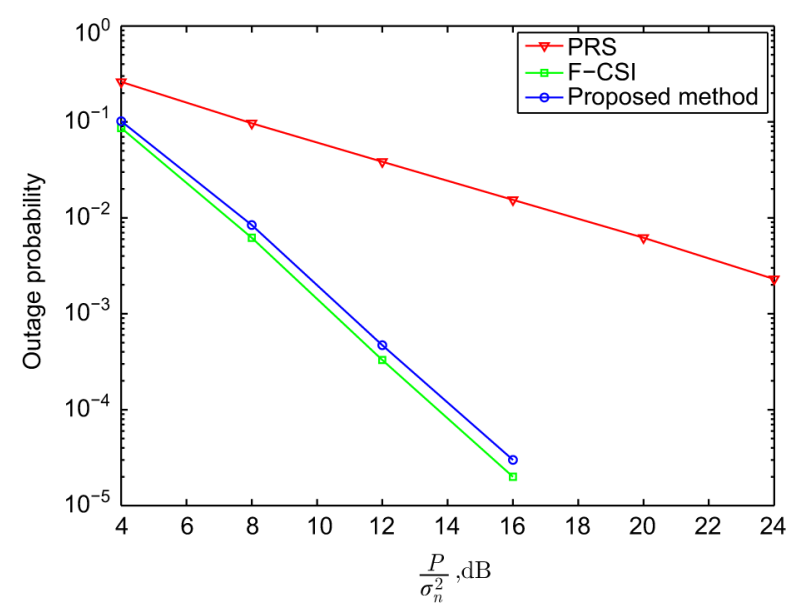

Fig. 8. Outage probability versus $\frac{P}{\sigma_{n}^{2}}$ for $R=3$ and non-identical channels.

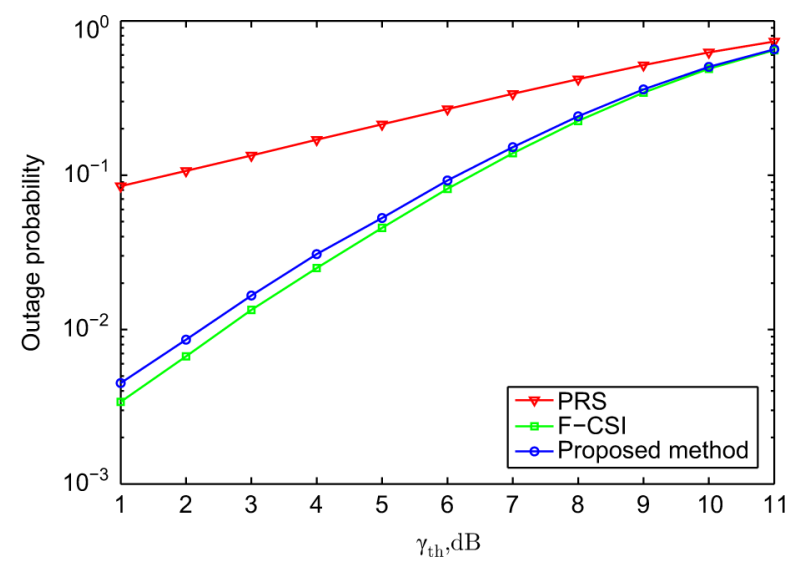

Fig. 9. Outage probability versus $\gamma_{\mathrm{th}}$ for $R=3$ and different source and relay powers.

\section{A. Results for $f_{j}=f, g_{j}=g, \forall j$}

In this subsection, we present theoretical and simulation results when channels of each hop are identically distributed, i.e., $f_{j}=f, g_{j}=g, \forall j$. For all results, we take $P_{\mathrm{S}}=P_{\mathrm{R}, j}=P$, and $c_{j, \mathrm{~d}}=c_{\mathrm{s}, j}=c, \forall j$. For the purpose of comparing theoretical results on diversity gain with simulations, we define a common average $\mathrm{SNR}$ as $\mathcal{S} \mathcal{N} \mathcal{R} \triangleq \frac{c P}{\sigma_{n}^{2}}$ and allow it to assume large values by varying $c P$ and keeping $\sigma_{n}^{2}=1$. Similar approach is used in other papers with AF relaying, e.g., [12] and [13], for the purpose of demonstrating the diversity gain.

The outage probabilities as a function of $\mathcal{S N} \mathcal{R}$, with different $\epsilon$ and selection methods, are shown in Figs. 1 and 2 for $R=2$ and $R=3$, respectively. It can be observed from both figures that there exists a fine agreement between theoretical and simulation results for the proposed method. Moreover, in both figures, the proposed scheme significantly outperforms PRS and, when $\epsilon$ is optimized, the proposed selection provides performance which is very close to that of the F-CSI scheme. Fig. 1 further shows that a full diversity gain of 2 can be achieved irrespective of the optimized $\epsilon$ or fixed $\epsilon$ (set to zero). However, Fig. 2 demonstrates that the proposed scheme does not achieve full diversity order of 3 when $\epsilon=0$ is taken. These observations verify our theoretical results and show that the optimization of $\epsilon$ is required in general for improving performance. For the proposed method and different $\epsilon$, the asymptotic and exact outage probabilities as a function of $\mathcal{S} \mathcal{N} \mathcal{R}$ are displayed in Figs. 3 and 4 for $R=2$ and $R=3$, respectively. It can be observed from these figures that the agreement between the asymptotic and exact outage probabilities in the high SNR region is very good.

In Figs. 5 and 6 , the respective outage probabilities versus $\gamma_{\mathrm{th}}$ are displayed for $R=2$ and $R=3$, and different selection methods, with different $\epsilon$. For both figures, we take $\mathcal{S} \mathcal{N} \mathcal{R}=16$ dB. As in case of Figs. 1 and 2, Figs. 5 and 6 show that the analytical and simulated outage probabilities match perfectly for the proposed method. These figures also show that the proposed selection method performs much better than the PRS scheme, especially for low to medium values of $\gamma_{\mathrm{th}}$. It can also be observed that the gap between the performance of the proposed scheme and that of the system having full CSI can be reduced by optimizing the value of $\epsilon$.

The performance of the three relay selection methods is displayed in Fig. 7 for $R=4$ and $R=5$. In this figure, for the proposed method, the value of $\epsilon$ is optimized using the asymptotic expression (30). It can be observed from Fig. 7 that the theoretical and simulated results of the proposed method exhibit good matching. This figure also shows that the proposed method significantly outperforms the PRS method, achieves full diversity and provides outage probability that is slightly inferior to that of the F-CSI method.

\section{B. Results for Arbitrary $f_{j}, g_{j}$}

This subsection provides simulation results when channels are non-identical, i.e., $f_{j}, g_{j}, \forall j$ assume arbitrary values. Taking the distance $\left(d_{0}\right)$ between the source and destination as a reference with $d_{0}=1$, the S-R and R-D distances are defined as $d_{\mathrm{s}, j}=\beta_{j} d_{0}$ and $d_{j, \mathrm{~d}}=\left(1-\beta_{j}\right) d_{0}$, where $\beta_{j} \leq 1$ are positive scalars. Furthermore, $c_{\mathrm{s}, j}$ and $c_{j, \mathrm{~d}}$ are given by $c_{\mathrm{s}, j}=d_{\mathrm{s}, j}^{-\eta}$ and $c_{j, \mathrm{~d}}=d_{j, \mathrm{~d}}^{-\eta}$, where $\eta$ is the path loss exponent. For all simulations of this subsection, we take $\eta=2$ and $\sigma_{n}^{2}=1$. Fig. 8 displays outage probability versus common average SNR $\frac{P}{\sigma^{2}}$, where $P=P_{\mathrm{S}}=P_{\mathrm{R}, j}$ is varied. In this figure, we take $R={ }^{n}$, $\beta_{1}=0.5, \beta_{2}=0.4, \beta_{3}=0.6$, and $\epsilon=0.2 \gamma_{\mathrm{th}}$. In Fig. 9, the outage probability versus $\gamma_{\text {th }}$ is shown for different transmit powers of source and relay nodes. In this figure, we take $R=3$, 
$\beta_{1}=\beta_{2}=\beta_{3}=0.5, P_{\mathrm{S}}=8 \mathrm{dBw}, P_{\mathrm{R}, 1}=5 \mathrm{dBw}, P_{\mathrm{R}, 2}=6$ $\mathrm{dBw}, P_{\mathrm{R}, 2}=7 \mathrm{dBw}$, and $\epsilon=0.2 \gamma_{\mathrm{th}}$. It can be observed from Figs. 8-9 that, as in the case with $f_{j}=f, g_{j}=g, \forall j$, the proposed method provides performance very close to that of the F-CSI method and significantly better than the PRS method for the cases with arbitrary $f_{j}, g_{j}, \forall j$. Fig. 8 shows that the proposed method also gives full diversity gain for the non-identical channels.

We now end this section with the following remarks. Although the proposed approach is slightly suboptimal (see Figs. 1-2 and 5-9) in terms of the outage probability, it is important to emphasize that the proposed scheme requires qualitatively less training overhead than the F-CSI method, e.g., by $(R-1) T_{\mathrm{fa}}$ (see Table I). The additional overhead of the F-CSI method increases linearly with $R$. On the other hand, the capacity of the relay selection scheme increases only logarithmically with respect to the SNR. By relating these two facts and bringing the advantage of the proposed method's reduced training overhead into a unified performance metric (e.g., outage capacity), we can verify that the proposed method outperforms F-CSI method with a high probability [6]. However, in order to demonstrate such a unified performance and compare different relay selection methods in a comprehensive and fair manner, we have to determine the exact training overhead required in each method. This exact overhead depends on several factors (e.g., channel conditions, average SNR, relay selection method) and, therefore, may be obtained only from extensive link level simulations of channel estimation algorithms. Since such task is out of the scope of this paper, we have compared the relay selection methods in terms of separate performance metrics, namely, the outage probability and qualitatively determined training overhead.

\section{CONCLUSIONS}

We have proposed a novel relay selection method for AF relay systems, which selects the relay with the maximum second-hop SNR from a subset of relays for which the S-R links are not in outage. The training overhead requirement of the proposed method is qualitatively compared with the PRS and OR schemes. The exact expressions of the outage probability are first derived for $R=2,3$, which are then extended to an arbitrary $R$. The asymptotic expressions of the outage probability are derived for $R=2,3$ by first taking $\epsilon=0$. After demonstrating that this choice of $\epsilon$ does not give full diversity for $R=3$, we derive asymptotic expression of the outage probability for an arbitrary $R$ by taking $\epsilon>0$, which is shown to yield full diversity. A method for optimizing $\epsilon$ is then proposed for further improving the overall performance of the proposed relay selection method. Simulation results confirm theoretical analysis and show that the new method significantly outperforms PRS and provides performance which is comparable to that of the method having full CSI. In a nut-shell, the developed scheme is shown to narrow the gap between the performance and complexity of the existing methods. The prospect of estimating the R-D channels at the candidate relays and sending the corresponding quantized channel estimates to the destination for relay selection is thought for future work as the complete analysis is beyond the scope of this paper.

\section{APPENDIX A}

\section{ProOF OF PROPOSITION 1}

We define $\tilde{r}_{3} \triangleq \operatorname{Pr}\left\{\gamma_{1} \leq \gamma_{\mathrm{th}} \mid \widetilde{\mathcal{B}}_{3}\right\} \operatorname{Pr}\left\{\widetilde{\mathcal{B}}_{3}\right\}$. Since $\gamma_{1}$ is independent of $b_{2}$ and $b_{3}$, we can write $\tilde{r}_{3}$ as

$$
\begin{aligned}
\tilde{r}_{3}= & \operatorname{Pr}\left\{\gamma_{1} \leq \gamma_{\mathrm{th}} \mid\left\{\alpha_{1, \mathrm{~d}} \geq \max \left(\alpha_{2, \mathrm{~d}}, \alpha_{3, \mathrm{~d}}\right), b_{1}=1,\right.\right. \\
& \left.\left.\left\{\alpha_{j, \mathrm{~d}}\right\}_{j=1}^{3} \geq \frac{\gamma_{\mathrm{th}}}{g}\right\}\right\} \operatorname{Pr}\left\{\widetilde{\mathcal{B}}_{3}\right\} \\
= & \operatorname{Pr}\left\{\gamma_{1} \leq \gamma_{\mathrm{th}} \mid\left\{\alpha_{1, \mathrm{~d}} \geq \max \left(\alpha_{2, \mathrm{~d}}, \alpha_{3, \mathrm{~d}}\right), b_{1}=1,\right.\right. \\
& \left.\left.\left\{\alpha_{j, \mathrm{~d}}\right\}_{j=1}^{3} \geq \frac{\gamma_{\mathrm{th}}}{g}\right\}\right\} \operatorname{Pr}\left\{\alpha_{1, \mathrm{~d}} \geq \max \left(\alpha_{2, \mathrm{~d}}, \alpha_{3, \mathrm{~d}}\right),\right. \\
& \left.b_{1}=1,\left\{\alpha_{j, \mathrm{~d}}\right\}_{j=1}^{3} \geq \frac{\gamma_{\mathrm{th}}}{g}\right\} \operatorname{Pr}\left\{b_{2}=1\right\} \operatorname{Pr}\left\{b_{3}=1\right\} \\
= & \operatorname{Pr}\left\{\gamma_{1} \leq \gamma_{\mathrm{th}}, \alpha_{1, \mathrm{~d}} \geq \max \left(\alpha_{2, \mathrm{~d}}, \alpha_{3, \mathrm{~d}}\right), b_{1}=1,\right. \\
& \left.\left\{\alpha_{j, \mathrm{~d}}\right\}_{j=1}^{3} \geq \frac{\gamma_{\mathrm{th}}}{g}\right\} \operatorname{Pr}\left\{b_{2}=1\right\} \operatorname{Pr}\left\{b_{3}=1\right\}
\end{aligned}
$$

where the second step is due to the fact that $\left[b_{2}=1, b_{3}=1\right]$ and $\left[\alpha_{1, \mathrm{~d}} \geq \max \left(\alpha_{2, \mathrm{~d}}, \alpha_{3, \mathrm{~d}}\right), b_{1}=1,\left\{\alpha_{j, \mathrm{~d}} \geq \frac{\gamma_{\mathrm{th}}}{g}\right\}_{j=1}^{3}\right]$ are statistically independent and the third step is due to the definition of conditional probability [25]. Since $b_{j}=1$ implies that $\alpha_{\mathrm{s}, j} \geq$ $\frac{\gamma_{\mathrm{th}}+\epsilon}{f}(j=2,3)$, we have $\operatorname{Pr}\left\{b_{2}=1\right\}=\operatorname{Pr}\left\{b_{3}=1\right\}=$ $\mathrm{e}^{-\frac{\left(\gamma_{\mathrm{th}}+\varepsilon\right)}{f}}$. Let the first probability term of $\tilde{r}_{3}$ in (33) be denoted by $r_{3}$. Substituting (4) into $\gamma_{1} \leq \gamma_{\text {th }}$ and integrating over the PDF of $\alpha_{\mathrm{s}, 1}, r_{3}$ can be expressed as

$$
\begin{gathered}
r_{3}=\int_{y=\frac{\gamma_{\mathrm{th}}+\mathrm{f}}{f}}^{\infty} \operatorname{Pr}\left\{\alpha_{1, \mathrm{~d}} \leq \frac{\gamma_{\mathrm{th}}(f y+1)}{g f y-g \gamma_{\mathrm{th}}},\left\{\alpha_{j, \mathrm{~d}}\right\}_{j=1}^{3} \geq \frac{\gamma_{\mathrm{th}}}{g}\right. \\
\left.\alpha_{1, \mathrm{~d}} \geq \alpha_{2, \mathrm{~d}}, \alpha_{1, \mathrm{~d}} \geq \alpha_{3, \mathrm{~d}}\right\} f_{\alpha_{\mathrm{s}, 1}}(y) d y
\end{gathered}
$$

where $f_{\alpha_{\mathrm{s}, 1}}(y)$ is the PDF of $\alpha_{\mathrm{s}, 1}$ and the event $\alpha_{1, \mathrm{~d}} \geq$ $\max \left\{\alpha_{j, \mathrm{~d}}\right\}_{j=2}^{3}$ is written as $\left[\alpha_{1, \mathrm{~d}} \geq \alpha_{2, \mathrm{~d}}, \alpha_{1, \mathrm{~d}} \geq \alpha_{3, \mathrm{~d}}\right]$. Since $\left\{\alpha_{j, \mathrm{~d}}\right\}_{j=1}^{3} \geq \frac{\gamma_{\mathrm{th}}}{g},(34)$ can be further expressed as

$$
\begin{aligned}
r_{3}= & \int_{v=\frac{\gamma_{\mathrm{th}}}{g}}^{\bar{v}} \int_{u=\frac{\gamma_{\mathrm{th}}}{g}}^{\bar{u}} \int_{y=\frac{\gamma_{\mathrm{th}}+\epsilon}{f}}^{\infty} \operatorname{Pr}\left\{\alpha_{1, \mathrm{~d}} \leq \frac{\gamma_{\mathrm{th}}(f y+1)}{g f y-g \gamma_{\mathrm{th}}},\right. \\
& \left.\alpha_{1, \mathrm{~d}} \geq u, \alpha_{1, \mathrm{~d}} \geq v\right\} f_{\alpha_{2, \mathrm{~d}}}(u) d u f_{\alpha_{3, \mathrm{~d}}}(v) d v f_{\alpha_{\mathrm{s}, 1}}(y) d y
\end{aligned}
$$

where $f_{\alpha_{2, \mathrm{~d}}}(u), f_{\alpha_{3, \mathrm{~d}}}(v)$ are the PDFs of $\alpha_{2, \mathrm{~d}}$ and $\alpha_{3, \mathrm{~d}}$, respectively, whereas, the respective upper limits of the variables $u$ and $v$ are $\bar{u}$ and $\bar{v}$. It is clear that the joint probability in (35) is not zero only for $\bar{u}=\bar{v} \leq \frac{\gamma_{\mathrm{th}}(f y+1)}{g f y-g \gamma_{\mathrm{th}}} \triangleq u_{\mathrm{yu}}$. Note that $\operatorname{Pr}\left\{\alpha_{1, \mathrm{~d}} \geq u, \alpha_{1, \mathrm{~d}} \geq v\right\}$ takes the following values

$$
\operatorname{Pr}\left\{\alpha_{1, \mathrm{~d}} \geq u, \alpha_{1, \mathrm{~d}} \geq v\right\}=\left\{\begin{array}{l}
\operatorname{Pr}\left\{\alpha_{1, \mathrm{~d}} \geq v\right\}, \text { for } v \geq u \\
\operatorname{Pr}\left\{\alpha_{1, \mathrm{~d}} \geq u\right\}, \text { for } u \geq v
\end{array} .\right.
$$

Due to (36), $r_{3}$ in (35) can be expressed as $r_{3}=I_{1}+I_{2}$, where

$$
I_{1}=\int_{y=\frac{\gamma_{\mathrm{th}}+\epsilon}{f}}^{\infty} \int_{u=\frac{\gamma_{\mathrm{th}}}{g}}^{u_{\mathrm{yu}}} \int_{v=u}^{u_{\mathrm{yu}}} \operatorname{Pr}\left\{\alpha_{1, \mathrm{~d}} \leq \frac{\gamma_{\mathrm{th}}(f y+1)}{g f y-g \gamma_{\mathrm{th}}},\right.
$$




$$
\begin{aligned}
\alpha_{1, \mathrm{~d}} & \geq v\} f_{\alpha_{3, \mathrm{~d}}}(v) d v f_{\alpha_{2, \mathrm{~d}}}(u) d u f_{\alpha_{\mathrm{s}, 1}}(y) d y \\
I_{2} & =\int_{y=\frac{\gamma_{\mathrm{th}}+\mathrm{\epsilon}}{f}}^{\infty} \int_{v=\frac{\gamma_{\mathrm{th}}}{g}}^{u_{\mathrm{yu}}} \int_{u=v}^{u_{\mathrm{yu}}} \operatorname{Pr}\left\{\alpha_{1, \mathrm{~d}} \leq \frac{\gamma_{\mathrm{th}}(f y+1)}{g f y-g \gamma_{\mathrm{th}}}\right. \\
\alpha_{1, \mathrm{~d}} & \geq u\} f_{\alpha_{2, \mathrm{~d}}}(u) d u f_{\alpha_{3, \mathrm{~d}}}(v) d v f_{\alpha_{\mathrm{s}, 1}}(y) d y
\end{aligned}
$$

Since $\left\{\alpha_{j, \mathrm{~d}}\right\}_{j=1}^{3}$ are identically distributed, it is clear from the limits of the integrals $I_{1}$ and $I_{2}$ that $I_{1}=I_{2}$. Thus, it is sufficient to solve either $I_{1}$ or $I_{2}$. With the variable substitution $\bar{y}=y-$ $\frac{\gamma_{\mathrm{th}}}{f}$ in $I_{1}$, we get $u_{\mathrm{yu}}=\frac{\gamma_{\mathrm{th}}}{g f}\left(f+\frac{\gamma_{\mathrm{th}}+1}{\bar{y}}\right)$. Then, solving the integration w.r.t. to the variables $u$ and $v$, and after some lengthy but straightforward steps, we obtain

$$
\begin{aligned}
I_{1}=\mathrm{e}^{-\frac{3 \gamma_{\text {th }}}{g}} & \int_{\frac{\mathrm{\epsilon}}{f}}^{\infty}\left\{\frac{1}{6}+\frac{1}{2}\left[\mathrm{e}^{-\frac{2 \gamma_{\text {th }} \bar{\gamma}_{\text {th }}}{g f \bar{y}}}-\mathrm{e}^{-\frac{\gamma_{\text {th }} \bar{\gamma}_{\text {th }}}{g f \bar{y}}}\right]\right. \\
& \left.-\frac{1}{6} \mathrm{e}^{-\frac{3 \gamma_{\text {th }} \bar{\gamma}_{\text {th }}}{g f \bar{y}}}\right\} f_{\alpha_{1, \mathrm{~d}}}\left(\bar{y}+\frac{\gamma_{\text {th }}}{f}\right) d \bar{y}
\end{aligned}
$$

where $\bar{\gamma}_{\text {th }}=\gamma_{\text {th }}+1$. Solving integration over $\bar{y}$ in (39), we obtain $I_{1}$. It follows that $r_{3}=I_{1}+I_{2}=2 I_{1}$. Thus, $r_{3}$ can be expressed as

$$
\begin{aligned}
& r_{3}=\mathrm{e}^{-\gamma_{\mathrm{th}}\left(\frac{3}{g}+\frac{1}{f}\right)}\left[\frac{1}{3} \mathrm{e}^{-\frac{\epsilon}{f}}+s_{2}-s_{1}-\frac{1}{3} s_{3}\right], \text { where }( \\
& s_{m}=\int_{\frac{\epsilon}{f}}^{\infty} \mathrm{e}^{-\bar{y}} \mathrm{e}^{-\frac{m \gamma_{\text {th }} \tilde{\gamma}_{\text {th }}}{g f \bar{y}}} d \bar{y}, m=[1,2,3] .
\end{aligned}
$$

For $\epsilon=0$, using (3.324.1) [26], the integral in (41) can be expressed as in (19), whereas, for $\epsilon>0$, this integral can be expressed in terms of Whittaker hypergeometric functions [26]. Using series expansion for an exponential function, we can re-express $s_{m}$ as

$$
s_{m}=\mathrm{e}^{-\frac{\epsilon}{f}}+\sum_{k=1}^{\infty}\left(-m \gamma_{\mathrm{th}} \bar{\gamma}_{\mathrm{th}}\right)^{k} \frac{(g f)^{-k}}{k !} \int_{\frac{\epsilon}{f}}^{\infty} \mathrm{e}^{-\bar{y}} \bar{y}^{-k} d \bar{y} .
$$

Applying (3.381.6) of [26], $s_{m}$ can be expressed as in (20) for $\epsilon>0$.

We define $\tilde{r}_{2} \triangleq \operatorname{Pr}\left\{\gamma_{1} \leq \gamma_{\mathrm{th}} \mid \widetilde{\mathcal{B}}_{2}\right\} \operatorname{Pr}\left\{\widetilde{\mathcal{B}}_{2}\right\}$. Since $\gamma_{1}$ is independent of $\left[b_{2}=1\right]$ and $\left[\alpha_{3, \mathrm{~d}}<\frac{\gamma_{\text {th }}}{g} \cup b_{3}=0\right]$, we can re-express $\tilde{r}_{2}$ as

$$
\begin{aligned}
\tilde{r}_{2}= & \operatorname{Pr}\left\{\gamma_{1} \leq \gamma_{\mathrm{th}} \mid\left\{\alpha_{1, \mathrm{~d}} \geq \alpha_{2, \mathrm{~d}}, b_{1}=1,\right.\right. \\
& \left.\left.\left\{\alpha_{j, \mathrm{~d}}\right\}_{j=1}^{2} \geq \frac{\gamma_{\mathrm{th}}}{g}\right\}\right\} \operatorname{Pr}\left\{\widetilde{\mathcal{B}}_{2}\right\} \\
= & \operatorname{Pr}\left\{\gamma_{1} \leq \gamma_{\mathrm{th}}, \alpha_{1, \mathrm{~d}} \geq \alpha_{2, \mathrm{~d}}, b_{1}=1,\left\{\alpha_{j, \mathrm{~d}}\right\}_{j=1}^{2} \geq \frac{\gamma_{\mathrm{th}}}{g}\right\} \\
& \times \operatorname{Pr}\left\{b_{2}=1\right\} \operatorname{Pr}\left\{\alpha_{3, \mathrm{~d}}<\frac{\gamma_{\mathrm{th}}}{g} \cup b_{3}=0\right\} .
\end{aligned}
$$

For convenience, let the first part of $\tilde{r}_{2}$ be defined as $r_{2} \triangleq \operatorname{Pr}\left\{\gamma_{1} \leq \gamma_{\mathrm{th}}, \alpha_{1, \mathrm{~d}} \geq \alpha_{2, \mathrm{~d}}, b_{1}=1,\left\{\alpha_{j, \mathrm{~d}}\right\}_{j=1}^{2} \geq \frac{\gamma_{\mathrm{th}}}{g}\right\}$. Note that the differences between $r_{2}$ and $r_{3}$ lie only in the facts that the event $\alpha_{1, \mathrm{~d}} \geq \max \left(\alpha_{2, \mathrm{~d}}, \alpha_{3, \mathrm{~d}}\right)$ of $r_{3}$ takes the form $\alpha_{1, \mathrm{~d}} \geq \alpha_{2, \mathrm{~d}}$ in $r_{2}$ and the event $\alpha_{3, \mathrm{~d}} \geq \frac{\gamma_{\text {th }}}{g}$ of $r_{3}$ does not appear in $r_{2}$. Thus, using similar steps of derivations as in the case with $r_{3}, r_{2}$ can be readily derived as

$$
r_{2}=\mathrm{e}^{-\frac{\gamma_{\text {th }}}{f}} \mathrm{e}^{-2 \frac{\gamma_{\mathrm{th}}}{g}}\left\{\frac{1}{2} \mathrm{e}^{-\frac{\epsilon}{f}}+\frac{1}{2} s_{2}-s_{1}\right\} .
$$

Let the second part of $\tilde{r}_{2}$ be defined as $\bar{r}_{2} \triangleq$ $\operatorname{Pr}\left\{b_{2}=1\right\} \operatorname{Pr}\left\{\alpha_{3, \mathrm{~d}}<\frac{\gamma_{\text {th }}}{g} \cup b_{3}=0\right\}$. Applying the fact that $\operatorname{Pr}\left\{x_{1} \cup x_{2}\right\}=\operatorname{Pr}\left\{x_{1}\right\}+\operatorname{Pr}\left\{x_{2}\right\}-\operatorname{Pr}\left\{x_{1}\right\} \operatorname{Pr}\left\{x_{2}\right\}$ for statistically independent $x_{1}$ and $x_{2}$, noting that $\operatorname{Pr}\left\{b_{2}=1\right\}=\operatorname{Pr}\left\{\alpha_{\mathrm{s}, 2} \geq \frac{\gamma_{\mathrm{th}}+\epsilon}{f}\right\}$, and $\alpha_{3, \mathrm{~d}}, \alpha_{\mathrm{s}, 2}$ and $\alpha_{\mathrm{s}, 3}$ are exponentially distributed, we readily get

$$
\bar{r}_{2}=\mathrm{e}^{-\frac{\gamma_{\text {th }}+\epsilon}{f}}\left[1-\mathrm{e}^{-\frac{\gamma_{\text {th }}}{g}} \mathrm{e}^{-\frac{\gamma_{\text {th }}+\epsilon}{f}}\right] .
$$

Thus, $\tilde{r}_{2}=r_{2} \bar{r}_{2}$ is obtained.

We define $\tilde{r}_{1} \triangleq \operatorname{Pr}\left\{\gamma_{1} \leq \gamma_{\mathrm{th}} \mid \widetilde{\mathcal{B}}_{1}\right\} \operatorname{Pr}\left\{\widetilde{\mathcal{B}}_{1}\right\}$. Since $\gamma_{1}$ is independent of $\left[\alpha_{j, \mathrm{~d}}<\frac{\gamma_{\mathrm{th}}}{g} \cup b_{j}=0\right]_{j=2}^{3}$, we get

$$
\begin{aligned}
\tilde{r}_{1}= & \operatorname{Pr}\left\{\gamma_{1} \leq \gamma_{\mathrm{th}} \mid\left[b_{1}=1, \alpha_{1, \mathrm{~d}} \geq \frac{\gamma_{\mathrm{th}}}{g}\right]\right\} \operatorname{Pr}\left\{\widetilde{\mathcal{B}}_{1}\right\} \\
= & \underbrace{\left.\operatorname{Pr}\left\{\gamma_{1} \leq \gamma_{\mathrm{th}}, b_{1}=1, \alpha_{1, \mathrm{~d}} \geq \frac{\gamma_{\mathrm{th}}}{g}\right]\right\}}_{r_{1}} \\
& \times \underbrace{\operatorname{Pr}\left\{\left[\alpha_{j, \mathrm{~d}} \leq \frac{\gamma_{\mathrm{th}}}{g} \cup b_{j}=0\right]_{j=2}^{3}\right\}}_{\bar{r}_{1}}
\end{aligned}
$$

which follows due to the statistical independence of $\left[b_{1}=1, \alpha_{1, \mathrm{~d}} \geq \frac{\gamma_{\mathrm{th}}}{g}\right] \quad$ and $\quad\left[\alpha_{j, \mathrm{~d}} \leq \frac{\gamma_{\mathrm{th}}}{g} \cup b_{j}=0\right]_{j=2}^{3} \quad$ and the definition of conditional probability. Since $b_{1}=1$ implies that $\alpha_{\mathrm{s}, 1} \geq \frac{\gamma_{\mathrm{th}}+\epsilon}{f}, r_{1}$ can be expressed as

$$
\begin{aligned}
r_{1}= & \int_{y=\frac{\gamma_{\mathrm{th}}+\epsilon}{f}}^{\infty} \operatorname{Pr}\left\{\alpha_{1, \mathrm{~d}} \leq \frac{\gamma_{\mathrm{th}}(f y+1)}{g f y-g \gamma_{\mathrm{th}}}, \alpha_{1, \mathrm{~d}} \geq \frac{\gamma_{\mathrm{th}}}{g}\right\} \\
& \times f_{\alpha_{\mathrm{s}, 1}}(y) d y .
\end{aligned}
$$

With similar steps as for $r_{2}$ and $\bar{r}_{2}, r_{1}$ and $\bar{r}_{1}$ can be given by

$$
\begin{aligned}
& r_{1}=\mathrm{e}^{-\gamma_{\mathrm{th}}\left(\frac{1}{g}+\frac{1}{f}\right)}\left(\mathrm{e}^{-\frac{\epsilon}{f}}-s_{1}\right), \\
& \bar{r}_{1}=\left[1-\mathrm{e}^{-\frac{\gamma_{\mathrm{th}}}{g}} \mathrm{e}^{-\frac{\gamma_{\mathrm{th}}+\epsilon}{f}}\right]^{2}
\end{aligned}
$$

where $s_{1}$ is given in (19)-(20). Finally, $r_{0}=\operatorname{Pr}\left\{\mathcal{E}_{3}\right\}$ is given by

$$
\begin{aligned}
r_{0} & =\operatorname{Pr}\left\{\left[\left[\alpha_{j, \mathrm{~d}} \leq \frac{\gamma_{\mathrm{th}}}{g}\right] \cup\left[\alpha_{\mathrm{s}, j} \leq \frac{\gamma_{\mathrm{th}}+\epsilon}{f}\right]\right]\right\} \\
& =\left[1-\mathrm{e}^{-\frac{\epsilon}{f}} \mathrm{e}^{-\gamma_{\mathrm{th}}\left(\frac{1}{g}+\frac{1}{f}\right)}\right]^{3} .
\end{aligned}
$$

This completes the proof of Proposition 1.

APPENDIX B

Proof of PRoposition 2

Consider a particular permutation $p_{1}$ of $\left\{u_{2}, \ldots, u_{l}\right\}$ where $\left\{u_{2} \geq u_{3} \geq, \ldots, \geq u_{l}\right\}$. For this case, (24) reduces to (see also (36)-(38)) 


$$
\begin{aligned}
r_{l}^{p_{1}}= & \int_{y=\frac{\gamma_{\mathrm{th}}+\varepsilon}{f}}^{\infty} \int_{u_{l}=\frac{\gamma_{\mathrm{th}}}{g}}^{\bar{u}} \cdots \int_{u_{3}=u_{4}}^{\bar{u}} \int_{u_{2}=u_{3}}^{\bar{u}} \operatorname{Pr}\left\{\alpha_{1, \mathrm{~d}} \leq\right. \\
& \left.\frac{\gamma_{\mathrm{th}}(f y+1)}{g f y-g \gamma_{\mathrm{th}}}, \alpha_{1, \mathrm{~d}} \geq u_{2}\right\} f_{\alpha_{2, \mathrm{~d}}}\left(u_{2}\right) d u_{2} \cdots \\
& \times f_{\alpha_{l, \mathrm{~d}}}\left(u_{l}\right) d u_{l} f_{\alpha_{\mathrm{s}, 1}}(y) d y .
\end{aligned}
$$

For solving (50), the result of the following lemma is used.

Lemma 1: Let $\left\{\alpha_{l, \mathrm{~d}}\right\}_{l=1}^{R}$ be independent and identically distributed exponential random variables with the probability density functions (PDFs) $f_{\alpha_{l, \mathrm{~d}}}\left(u_{l}\right)=\mathrm{e}^{-u_{l}}, \forall l$. Then, the integral

$$
\begin{aligned}
\tilde{I}= & \int_{u_{R}=\frac{\gamma_{\mathrm{th}}}{g}}^{\bar{u}} \cdots \int_{u_{3}=u_{4}}^{\bar{u}} \int_{u_{2}=u_{3}}^{\bar{u}} \operatorname{Pr}\left\{\alpha_{1, \mathrm{~d}} \leq \bar{u}, \alpha_{1, \mathrm{~d}} \geq u_{2}\right\} \\
& \times f_{\alpha_{2, \mathrm{~d}}}\left(u_{2}\right) d u_{2} f_{\alpha_{3, \mathrm{~d}}}\left(u_{3}\right) d u_{3} \cdots f_{\alpha_{R, \mathrm{~d}}}\left(u_{R}\right) d u_{R}
\end{aligned}
$$

can be expressed as

$$
\tilde{I}=\sum_{m=0}^{R} \frac{(-1)^{m}}{m !(R-m) !} \mathrm{e}^{-m \bar{u}} \mathrm{e}^{-(R-m) \frac{\gamma_{\mathrm{th}}}{g}} .
$$

Proof: Since $f_{\alpha_{1, \mathrm{~d}}}\left(u_{1}\right)=\mathrm{e}^{-u_{1}}$, it is easy to see that $\operatorname{Pr}\left\{\alpha_{1, \mathrm{~d}} \leq \bar{u}, \alpha_{1, \mathrm{~d}} \geq u_{2}\right\}=\mathrm{e}^{-u_{2}}-\mathrm{e}^{-\bar{u}}$. Consequently, the first inner integral of $\tilde{I}$ can be expressed as

$$
\begin{aligned}
\tilde{I}_{1} & =\int_{u 2=u 3}^{\bar{u}}\left(\mathrm{e}^{-u_{2}}-\mathrm{e}^{-\bar{u}}\right) f_{\alpha_{2, \mathrm{~d}}}\left(u_{2}\right) d u_{2} \\
& =\frac{1}{2} \mathrm{e}^{-2 \bar{u}}+\frac{1}{2} \mathrm{e}^{-2 u_{3}}-\mathrm{e}^{-\bar{u}} \mathrm{e}^{-u_{3}} .
\end{aligned}
$$

Using $\tilde{I}_{1}$, the second inner integral of $\tilde{I}$ can be given by

$$
\begin{gathered}
\tilde{I}_{2}=\int_{u 3=u 4}^{\bar{u}} \tilde{I}_{1} f_{\alpha_{3, \mathrm{~d}}}\left(u_{3}\right) d u_{3}=-\frac{1}{3 !} \mathrm{e}^{-3 \bar{u}}+\frac{1}{3 !} \mathrm{e}^{-3 u_{4}} \\
+\frac{1}{2 !} \mathrm{e}^{-2 \bar{u}} \mathrm{e}^{-u_{4}}-\frac{1}{2 !} \mathrm{e}^{-\bar{u}} \mathrm{e}^{-2 u_{4}} .
\end{gathered}
$$

In a similar way, we obtain

$$
\begin{aligned}
\tilde{I}_{3}= & \int_{u 4=u 5}^{\bar{u}} \tilde{I}_{2} f_{\alpha_{4, \mathrm{~d}}}\left(u_{4}\right) d u_{4}=\frac{1}{4 !} \mathrm{e}^{-4 \bar{u}}+\frac{1}{4 !} \mathrm{e}^{-4 u_{5}} \\
& -\frac{1}{3 !} \mathrm{e}^{-3 \bar{u}} \mathrm{e}^{-u_{5}}+\frac{1}{2 ! 2 !} \mathrm{e}^{-2 \bar{u}} \mathrm{e}^{-2 u_{5}}-\frac{1}{3 !} \mathrm{e}^{-\bar{u}} \mathrm{e}^{-3 u_{5}} .
\end{aligned}
$$

Therefore, following the rule of mathematical induction, we can express

$$
\tilde{I}=\sum_{m=0}^{R} \frac{(-1)^{m}}{m !(R-m) !} \mathrm{e}^{-m \bar{u}} \mathrm{e}^{-(R-m) u_{R+1}} .
$$

From (54)-(56) and the definition of $\tilde{I}$ in (51), it is clear that $u_{R+1}=u_{R}=\frac{\gamma_{\mathrm{th}}}{g}$. Thus, the desired expression (52) is obtained. This completes the proof of Lemma 1.

With the help of (51), $r_{l}^{\mathrm{p} l}$ can be expressed as

$$
r_{l}^{p_{1}}=\int_{y=\frac{\gamma_{\mathrm{th}}+\mathrm{\epsilon}}{f}}^{\infty} \tilde{I} f_{\alpha_{\mathrm{s}, 1}}(y) d y .
$$

Substituting $\tilde{I}$ from (52) into (57), we obtain

$$
r_{l}^{p_{1}}=\mathrm{e}^{-\gamma_{\mathrm{th}}\left(\frac{1}{f}+\frac{l}{g}\right)} \sum_{m=0}^{l} \frac{(-1)^{m}}{m !(l-m) !} s_{m}
$$

where $s_{0}^{(\epsilon>0)}=\mathrm{e}^{-\frac{\epsilon}{f}}$ and $s_{0}^{(\epsilon=0)}=1$. There are $(l-1)$ ! possible permutations (like $p_{1}$ ) for $\left\{u_{2}, \ldots, u_{l}\right\}$. Due to the fact that all $\alpha_{l, \mathrm{~d}}$ and $\alpha_{\mathrm{s}, l}$ are identically distributed, the integrals for all other permutations equal $r_{l}^{p_{1}}$. It follows that $r_{l}=(l-1) ! r_{l}^{p_{1}}$, which means that we obtain the expression (25) for $r_{l}$.

\section{APPENDIX C}

PROOF OF PROPOSITION 3

After some straightforward steps, (21) can be expressed as

$$
\begin{gathered}
P_{\mathrm{o}, 2}^{(\epsilon=0)}=\mathrm{e}^{-\gamma_{\mathrm{th}}\left(\frac{1}{g}+\frac{1}{f}\right)}\left\{\mathrm{e}^{-\gamma_{\mathrm{th}}\left(\frac{1}{g}+\frac{1}{f}\right)}\left(s_{2}^{(\epsilon=0)}-1\right)-2 s_{1}^{(\epsilon=0)}+2\right\} \\
+\left[1-\mathrm{e}^{-\gamma_{\mathrm{th}}\left(\frac{1}{g}+\frac{1}{f}\right)}\right]^{2} .
\end{gathered}
$$

For small $x(x \rightarrow 0), K_{1}(x)$ has the series form [22]

$$
\begin{aligned}
K_{1}(x)=\frac{1}{x} & +\frac{x}{4}\left(2 \gamma_{c}-1+\frac{1}{8}\left(2 \gamma_{c}-\frac{5}{2}\right) x^{2}+\mathcal{O}(x)\right) \\
& +\frac{x}{2} \log \left(\frac{x}{2}\right)\left(1+\frac{x^{2}}{8}+\mathcal{O}(x)\right)
\end{aligned}
$$

where $\gamma_{\mathrm{c}}$ is Euler's constant [26] and $\mathcal{O}(x)$ denotes higher order terms of $x$. Using (60), $x K_{1}(x)$ can be approximated for $x \rightarrow 0$ as

$$
x K_{1}(x) \approx 1+\frac{x^{2}}{4}\left(2 \gamma_{c}-1\right)+\frac{x^{2}}{2} \log \left(\frac{x}{2}\right) .
$$

For simplicity, let us define $\tilde{r} \triangleq \mathrm{e}^{-\gamma_{\text {th }}\left(\frac{1}{g}+\frac{1}{f}\right)}\left(s_{2}^{(\epsilon=0)}-1\right)-$ $2 s_{1}^{(\epsilon=0)}+2$ in (59). Applying (61) to $s_{1}^{(\epsilon=0)}$ and $s_{2}^{(\epsilon=0)}$, where $x$ takes values of $2 \sqrt{\frac{\gamma_{\mathrm{th}}\left(\gamma_{\mathrm{th}}+1\right)}{g f}}$ and $2 \sqrt{\frac{2 \gamma_{\mathrm{th}}\left(\gamma_{\mathrm{th}}+1\right)}{g f}}$, respectively, we can express $\tilde{r}$ asymptotically as

$$
\begin{aligned}
& \tilde{r} \approx \frac{2 \gamma_{\mathrm{th}}\left(\gamma_{\mathrm{th}}+1\right)}{g f}\left\{\left(2 \gamma_{\mathrm{c}}-1\right)\left(\mathrm{e}^{-\gamma_{\mathrm{th}}\left(\frac{1}{g}+\frac{1}{f}\right)}-1\right)+\right. \\
& \mathrm{e}^{-\gamma_{\mathrm{th}}\left(\frac{1}{g}+\frac{1}{f}\right)} \log \left(2 \gamma_{\mathrm{th}}\left(\gamma_{\mathrm{th}}+1\right)(g f)^{-1}\right) \\
& \left.-\log \left(\gamma_{\mathrm{th}}\left(\gamma_{\mathrm{th}}+1\right)(g f)^{-1}\right)\right\} .
\end{aligned}
$$

Substituting (62) into (59) and using the fact that $e^{-x} \approx 1-$ $x+x^{2} / 2$ for small $x$, we obtain

$$
\begin{aligned}
& P_{\mathrm{o}, 2}^{(\epsilon=0)} \approx\left(\tilde{x}-\frac{\tilde{x}^{2}}{2}\right)^{2}+2 \gamma_{\mathrm{th}}\left(\gamma_{\mathrm{th}}+1\right)(g f)^{-1}\{\log (2)+ \\
& \left.\left(-\tilde{x}+\frac{\tilde{x}^{2}}{2}\right)\left\{2 \gamma_{\mathrm{c}}-1+\log \left(2 \gamma_{\mathrm{th}}\left(\gamma_{\mathrm{th}}+1\right)(g f)^{-1}\right)\right\}\right\}
\end{aligned}
$$

where $\tilde{x} \triangleq \gamma_{\mathrm{th}}\left(\frac{1}{e}+\frac{1}{f}\right)$. Emphasizing the term with the lowest order of $\frac{1}{g f}$, (63) can be written as (26). 


\section{APPENDIX D \\ ProOF OF PROPOSITION 4}

For $\epsilon=0$, the outage probability (17) is expressed as

$$
\begin{aligned}
P_{\mathrm{o}, 3}^{(\epsilon=0)}= & 3 \mathrm{e}^{-3 \gamma_{\mathrm{th}}\left(\frac{1}{g}+\frac{1}{f}\right)}\left\{\frac{1}{3}+s_{2}^{(\epsilon=0)}-s_{1}^{(\epsilon=0)}-\frac{1}{3} s_{3}^{(\epsilon=0)}\right\}+ \\
& 6 \mathrm{e}^{-2 \gamma_{\mathrm{th}}\left(\frac{1}{g}+\frac{1}{f}\right)}\left[0.5 s_{2}^{(\epsilon=0)}-s_{1}^{(\epsilon=0)}+0.5\right] \\
& \times\left[1-\mathrm{e}^{-\gamma_{\mathrm{th}}\left(\frac{1}{g}+\frac{1}{f}\right)}\right]+\left[1-\mathrm{e}^{-\gamma_{\mathrm{th}}\left(\frac{1}{g}+\frac{1}{f}\right)}\right]^{2} \\
& \left\{3\left(1-s_{1}^{(\epsilon=0)}\right) \mathrm{e}^{-\gamma_{\mathrm{th}}\left(\frac{1}{g}+\frac{1}{f}\right)}+1-\mathrm{e}^{-\gamma_{\mathrm{th}}\left(\frac{1}{g}+\frac{1}{f}\right)}\right\} .
\end{aligned}
$$

Using the asymptotic result (61) for $s_{m}^{(\epsilon=0)}, m=1,2,3$ in (64) and after some straightforward derivations, we obtain

$$
\begin{aligned}
\frac{1}{3}+s_{2}^{(\epsilon=0)}-s_{1}^{(\epsilon=0)}-\frac{1}{3} s_{3}^{(\epsilon=0)} \approx \gamma_{\mathrm{th}}\left(\gamma_{\mathrm{th}}+1\right)(g f)^{-1} \\
\times(2 \log (2)-\log (3)) .
\end{aligned}
$$

Similarly, with the help of (61), we obtain

$$
\begin{aligned}
0.5 s_{2}^{(\epsilon=0)}-s_{1}^{(\epsilon=0)}+0.5 \approx & \gamma_{\mathrm{th}}\left(\gamma_{\mathrm{th}}+1\right)(g f)^{-1} \log (2), \\
1-s_{1}^{(\epsilon=0)} \approx & -\gamma_{\mathrm{th}}\left(\gamma_{\mathrm{th}}+1\right)(g f)^{-1}\left\{\left(2 \gamma_{\mathrm{c}}-1\right)\right. \\
& \left.+\log \left(\gamma_{\mathrm{th}}\left(\gamma_{\mathrm{th}}+1\right)(g f)^{-1}\right)\right\} .
\end{aligned}
$$

Using (65) and (66), and the fact that $e^{-x} \approx 1-x$ for small $x$, we can approximate (64) as

$$
\begin{aligned}
P_{\mathrm{o}, 3}^{(\epsilon=0)} \approx & \gamma_{\mathrm{th}}\left(\gamma_{\mathrm{th}}+1\right)(g f)^{-1}\{3(1-3 \tilde{x})(2 \log (2)-\log (3)) \\
& +6(1-2 \tilde{x}) \log (2) \tilde{x}-3(1-\tilde{x})\left(\left(2 \gamma_{\mathrm{c}}-1\right)\right. \\
& \left.\left.+\log \left(\gamma_{\mathrm{th}}\left(\gamma_{\mathrm{th}}+1\right)(g f)^{-1}\right)\right) \tilde{x}^{2}\right\}+\tilde{x}^{3}
\end{aligned}
$$

Highlighting the terms with the lowest order of $\frac{1}{g f}$, we get the asymptotic expression of (27).

\section{APPENDIX E}

\section{PROOF OF PROPOSITION 5}

Notice that the expression for $s_{m}^{(\epsilon>0)}(20)$ can be given by

$$
\begin{gathered}
s_{m}^{(\epsilon>0)}=\mathrm{e}^{-\frac{\epsilon}{f}}+\left(-m \tilde{\gamma}_{\mathrm{th}}\right) \mathrm{e}^{-\frac{\epsilon}{2 f}} g^{-1}(\epsilon f)^{-\frac{1}{2}} W_{-\frac{1}{2}, 0}\left(\frac{\epsilon}{f}\right) \\
+\sum_{k=2}^{\infty} \frac{\left(-m \tilde{\gamma}_{\mathrm{th}}\right)^{k}}{k !} \mathrm{e}^{-\frac{\epsilon}{2 f}} g^{-k}(\epsilon f)^{-\frac{k}{2}} W_{-\frac{k}{2}, \frac{1-k}{2}}\left(\frac{\epsilon}{f}\right),
\end{gathered}
$$

whereas $r_{l}$ (25) for $\epsilon>0$, can be expressed as

$$
r_{l}=\mathrm{e}^{-\gamma_{\mathrm{th}}\left(\frac{1}{f}+\frac{l}{g}\right)}(l-1) !\left\{\frac{1}{l !} \mathrm{e}^{-\frac{\epsilon}{f}}+\sum_{m=1}^{l} \frac{(-1)^{m} s_{m}^{(\epsilon>0)}}{m !(l-m) !}\right\} .
$$

Substituting $s_{m}^{(\epsilon>0)}$ from (68) into (69), and noting that

$$
\sum_{m=1}^{l} \frac{(-1)^{m}}{m !(l-m) !}=-\frac{1}{l !}
$$

we get

$$
\begin{aligned}
r_{l}= & \mathrm{e}^{-\gamma_{\mathrm{th}}\left(\frac{1}{f}+\frac{l}{g}\right)}(l-1) !\left\{\sum_{m=1}^{l} \frac{(-1)^{m+1} m^{1}}{m !(l-m) !} \tilde{\gamma}_{\mathrm{th}} \mathrm{e}^{-\frac{\epsilon}{2 f}}\right. \\
& g^{-1}(\epsilon f)^{-\frac{1}{2}} W_{-\frac{1}{2}, 0}\left(\frac{\epsilon}{f}\right)+\sum_{m=1}^{l} \sum_{k=2}^{\infty} \frac{(-1)^{m+k} m^{k}}{m !(l-m) !} \\
& \left.\times \frac{\tilde{\gamma}_{\mathrm{th}}^{k}}{k !} \mathrm{e}^{-\frac{\epsilon}{2 f}} g^{-k}(\epsilon f)^{-\frac{k}{2}} W_{-\frac{k}{2}, \frac{1-k}{2}}\left(\frac{\epsilon}{f}\right)\right\} .
\end{aligned}
$$

At high SNR, i.e., for $f \rightarrow \infty$ with a given $\epsilon, \frac{\epsilon}{f} \rightarrow 0$. We can then obtain the asymptotic series expansion of $W_{-\frac{k}{2}, \frac{1-k}{2}}\left(\frac{\epsilon}{f}\right)$. For this purpose, we first state the following series for $W_{\lambda, \mu}(z)(z \rightarrow 0)$ with the parameters $\lambda$ and $\mu$ [22]:

$$
\begin{aligned}
W_{\lambda, \mu}(z) \approx & -\frac{z^{\mu+\frac{1}{2}}}{\Gamma\left(\mu-\lambda+\frac{1}{2}\right)}\left\{\frac{(-z)^{-2 \mu} \log (z)}{(-2 \mu) !}(1+\mathcal{O}(z))\right. \\
& \left.-\frac{(-2 \mu-1) !}{\left(\mu-\lambda+\frac{1}{2}\right)-2 \mu}(1+\mathcal{O}(z))\right\},-2 \mu \in \mathcal{N}^{+}, \\
W_{\lambda, 0}(z) \approx- & \frac{z^{\frac{1}{2}}}{\Gamma\left(\frac{1}{2}-\lambda\right)}\left\{\log (z)(1+\mathcal{O}(z))+\Psi\left(\frac{1}{2}-\lambda\right)\right. \\
& \left.+2 \gamma_{c}+\mathcal{O}(z)\right\}
\end{aligned}
$$

where $\mathcal{N}^{+}$is the set of positive integers, the symbol $(x)_{n}$ stands for Prochammer symbol with positive integer $n$, i.e., $(x)_{n}=$ $x(x+1) \cdots(x+n-1)$, and $\Psi(x)$ denotes Polygamma function where $\Psi(x)=\sum_{k=1}^{\infty}\left(\frac{1}{k}-\frac{1}{k+x-1}\right)-\gamma_{\mathrm{c}}$ and $\gamma_{\mathrm{c}}$ is Euler's constant [26]. Using (72) and (73) for $W_{-\frac{k}{2}, \frac{1-k}{2}}\left(\frac{\epsilon}{f}\right)$, where $k-1 \in \mathcal{N}^{+}$, we obtain

$$
\begin{aligned}
(\epsilon f)^{-\frac{k}{2}} W_{-\frac{k}{2}, \frac{1-k}{2}}\left(\frac{\epsilon}{f}\right) \approx & \left\{\frac{(-1)^{k}}{(k-1) !} \log \left(\frac{\epsilon}{f}\right) f^{-k}+(k-2)\right. \\
& \left.\epsilon^{1-k} f^{-1}\right\}\left(1+\mathcal{O}\left(\frac{\epsilon}{f}\right)\right), \quad(74) \\
(\epsilon f)^{-\frac{1}{2}} W_{-\frac{1}{2}, 0}\left(\frac{\epsilon}{f}\right) \approx & -f^{-1}\left\{\log \left(\frac{\epsilon}{f}\right)\left(1+\mathcal{O}\left(\frac{\epsilon}{f}\right)\right)\right. \\
& \left.+\Psi(1)+2 \gamma_{c}+\mathcal{O}\left(\frac{\epsilon}{f}\right)\right\} .
\end{aligned}
$$

Substituting (74)-(75) into (71) and noting that $\mathrm{e}^{-x} \approx 1-x$ for small $x$, we get

$$
\begin{aligned}
r_{l} \approx & (l-1) !\left\{-\tilde{\gamma}_{\mathrm{th}}(g f)^{-1}\left(\log \left(\frac{\epsilon}{f}\right)+\gamma_{\mathrm{c}}\right) \sum_{m=1}^{l} \frac{(-1)^{m+1} m^{1}}{m !(l-m) !}\right. \\
& +\sum_{m=1}^{l} \sum_{k=2}^{\infty} \frac{(-1)^{m+k} m^{k}}{m !(l-m) !} \frac{\tilde{\gamma}_{\mathrm{th}}^{k}}{k !}
\end{aligned}
$$




$$
\left.\left[\frac{(-1)^{k}}{(k-1) !} \log \left(\frac{\epsilon}{f}\right)(g f)^{-k}+(k-2) \epsilon^{1-k} g^{-k} f^{-1}\right]\right\}
$$

where, for the last equation, we used the fact $\Psi(1)=-\gamma_{\mathrm{c}}$. Now, we proceed to analyze the dominating terms of (76) at high SNR for specific values of $l$. We then generalize these results for an arbitrary $l$.

It can be readily shown that $\sum_{m=1}^{l} \frac{(-1)^{m+1} m^{1}}{m !(l-m) !}$ takes value of 1 for $l=1$ and zeros for all $l>1$. Therefore, $r_{1}$ can be further approximated as

$$
r_{1} \approx-\tilde{\gamma}_{\mathrm{th}}(g f)^{-1}\left(\log \left(\frac{\epsilon}{f}\right)+\gamma_{\mathrm{c}}\right) .
$$

After expanding (76) for $l=2$, the terms that dominate $r_{2}$ at high SNR turn to be weighted by $\sum_{m=1}^{l} \frac{(-1)^{m+2} m^{2}}{m !(l-m) !}$ and $\sum_{m=1}^{l} \frac{(-1)^{m+3} m^{3}}{m !(l-m) !}$, which respectively, take values of 1 and -3 . It follows that $r_{2}$ can be expressed as

$$
r_{2} \approx \frac{1}{2} \tilde{\gamma}_{\mathrm{th}}^{2} \log \left(\frac{\epsilon}{f}\right)(g f)^{-2}-\frac{1}{2} \tilde{\gamma}_{\mathrm{th}}^{3} \epsilon^{-2} g^{-3} f^{-1} .
$$

For $l \geq 3$, the dominating term in (76) can be shown to be weighted by $\sum_{m=1}^{l} \frac{(-1)^{m+l} m^{l}}{m !(l-m) !}$ which takes value of 1 . Thus, we get

$$
r_{l} \approx \frac{1}{l} \tilde{\gamma}_{\mathrm{th}}^{l} \epsilon^{-(1-l)} g^{-l} f^{-1}(l-2), l \geq 3
$$

With these results, we complete the proof for Proposition 5.

\section{REFERENCES}

[1] A. S. Avestimehr and D. N. C. Tse, "Outage capacity of the fading relay channel in the low SNR regime," IEEE Trans. Inf. Theory, vol. 53, no. 4, pp. 1401-1415, Apr. 2007.

[2] J. N. Laneman and G. W. Wornell, "Exploiting distributed spatial diversity in wireless networks," in Proc. Allerton Conf. Comm., Cont. Comp., Monticello, IL, USA, Oct. 2000.

[3] L. Zheng and D. N. C. Tse, "Diversity and multiplexing: A fundamental tradeoff in multiple-antenna channels," IEEE Trans. Inf. Theory, vol. 49, no. 5, pp. 1073-1096, May 2003.

[4] I. F. Akyildiz, D. M. Gutierrez-Estevez, and E. C. Reyes, "The evolution to 4G cellular systems: LTE-advanced," Phys. Commun., vol. 3, pp. $217-244,2010$.

[5] B. K. Chalise and L. Vandendorpe, "Optimization of MIMO relays for multipoint-to-multipoint communications: Nonrobust and robust designs," IEEE Trans. Signal Process., vol. 58, no. 12, pp. 6355-6368, Dec. 2010.

[6] B. K. Chalise, Y. D. Zhang, and M. G. Amin, "Local CSI based selection beamforming for AF MIMO relay system with direct link," IEEE Commun. Lett., vol. 16, no. 5, pp. 622-625, May 2012.

[7] A. Blestas, A. Khisti, D. P. Reed, and A. Lippman, "A simple cooperative diversity method based on network path selection," IEEE J. Sel. Areas Commun, vol. 24, no. 3, pp. 659-672, Mar. 2006.

[8] Y. Zhao, R. Adve, and T. J. Lim, "Symbol error rate of selection amplify-and-forward relay systems," IEEE Commun. Lett., vol. 10, no. 11, pp. 757-759, Nov. 2006.

[9] Y. Jing and H. Jafarkhani, "Single and multiple relay selection schemes and their achievable diversity order," IEEE Trans. Wireless Commun., vol. 7, pp. 1414-1423, Mar. 2009.

[10] F. Gao, T. Cui, and A. Nallanathan, "On channel estimation and optimal training design for amplify and forward relay networks," IEEE Trans. Wireless Commun., vol. 7, no. 5, pp. 1907-1916, May 2008.

[11] C. S. Patel and G. L. Stuber, "Channel estimation for amplify and forward relay based cooperation diversity systems," IEEE Trans. Wireless Commun., vol. 6, no. 6, pp. 2348-2356, Jan. 2007.
[12] I. Krikidis, J. Thompson, S. McLaughlin, and N. Goertz, "Amplifyand-forward with partial relay selection," IEEE Commun. Lett., vol. 12, no. 4, pp. 235-237, Apr. 2008.

[13] J.-B. Kim and D. Kim, "Comparison of tightly power-constrained performances for opportunistic amplify-and-forward relaying with partial or full channel information," IEEE Commun. Lett., vol. 13, no. 2, pp. 100-102, Feb. 2009

[14] D. B. de Costa and S. Aissa, "End-to-end performance of dual-hop semi-blind relaying systems with partial relay selection," IEEE Trans. Wireless Commun., vol. 8, no. 8, pp. 4306-4315, Aug. 2009.

[15] B. K. Chalise, L. Vandendorpe, Y. D. Zhang, and M. G. Amin, "Local CSI based selection beamforming for amplify-and-forward MIMO relay networks," IEEE Trans. Signal Process., vol. 60, no. 5, pp. 2433-2446, May 2012.

[16] B. K. Chalise, Y. D. Zhang, and M. G. Amin, "A novel method for partial relay selection in amplify-and-forward relay systems," in Proc. IEEE Globecom 2012, Anaheim, CA, Dec. 2012, pp. 4695-4700.

[17] E. Beres and R. Adve, "On selection cooperation in distributed networks," Proc. 40th Annual Inform. Sci. Sys., pp. 1056-1061, Mar. 2006.

[18] D. S. Michalopoulos and G. K. Karagiannidis, "Performance analysis of single relay selection in Rayleigh fading," IEEE Trans. Wireless Commun., vol. 7, no. 10, pp. 3718-3724, Oct. 2008.

[19] G. C. Alexandropoulos, A. Papadogiannis, and K. Berberidis, "Performance analysis of cooperative networks with relay selection over Nakagami- $m$ fading channels," IEEE Signal Process. Lett., vol. 17, no. 5, pp. 441-444, May 2010.

[20] A. S. Ibrahim, A. K. Sadek, W. Su, and K. J. R. Liu, "Cooperative communications with relay-selection: When to cooperate and whom to cooperate with?," IEEE Trans. Wireless Commun., vol. 7, no. 7, pp. 2814-2827, Jul. 2008.

[21] S. Buzzi, M. Lops, and S. Sardellitti, "Performance of iterative data detection and channel estimation for single-antenna and multiple-antennas wireless communications," IEEE Trans. Veh. Technol., vol. 53, no. 4, pp. 1085-1104, Jul. 2004.

[22] [Online]. Available: http://functions.wolfram.com/.

[23] M. O. Hasna and M.-S. Alouini, "End-to-end performance of transmission systems with relays over Rayleigh-fading channels," IEEE Trans. Wireless Commun., vol. 2, no. 6, pp. 1126-1131, Nov. 2003.

[24] M. Biguesh and A. B. Gershman, "Training-based MIMO channel estimation: A study of estimator tradeoffs and optimal training signals," IEEE Trans. Signal Process., vol. 54, no. 3, pp. 884-893, Mar. 2006.

[25] A. Papoulis, Probability, Random Variables, and Stochastic Processes. New York, NY, USA: McGraw-Hill, 1991.

[26] I. S. Gradshteyn and I. M. Ryzhik, Table of Integrals, Series, and Products. New York, NY, USA: Academic, 2000.

[27] S. Boyd and L. Vandenberghe, Convex Optimization. Cambridge, U.K.: Cambridge Univ. Press, 2004.

[28] V. Stencel, A. Müller, and P. Frank, "Lte advanced: A further evolutionary step for next generation mobile networks," in Proc. 20th Int. Conf. Radioelektronika, Apr. 2010.

[29] J. Cho and Z. J. Haas, "On the throughput enhancement of the downstream channel in cellular radio networks through multihop relaying," IEEE J. Sel. Areas Commun., vol. 22, no. 7, pp. 1206-1219, Sep. 2004.

[30] D. Soldani, Multi-hop relay network [Online]. Available: http://cdn. intechopen.com/pdfs/9483/InTech-Multi_hop_relay_networks.pdf.

[31] H. Feng and L. J. Cimini, Jr, "On optimum relay deployment in a multihop linear network with cooperation," in Proc. IEEE MiLCOM, 2012.

[32] B. Gui, L. Dai, and L. J. Cimini, Jr, "Routing strategies in multihop cooperative networks," IEEE Trans. Wireless Commun., vol. 8, no. 2, pp. 843-855, Feb. 2009.

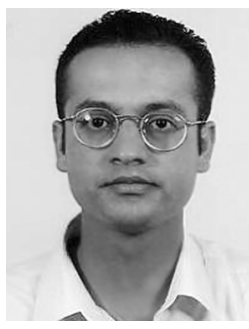

Batu Krishna Chalise (SM'11) received the B. E. degree in electronics engineering from Tribhuvan University, Kathmandu, in 1998 and the M.S. and Ph.D. degrees in electrical engineering from the University of Duisburg-Essen, Duisburg, Germany, in 2001 and 2006, respectively.

In 1999, he was a lecturer with the Institute of Engineering, Kathmandu. For a short period in 2001, he was a Researcher with the Fraunhofer-Institute of Microelectronic Circuits and Systems (IMS), Duisburg. From 2002 to 2006, he was a Research Assistant with the Department of Communication Systems, University of DuisburgEssen, where his Ph.D. research was supported by a grant from the Ministry of Education and Science of North Rhein-Westphalia (NRW), Germany. He was 
a Postdoctoral Researcher with the Communication and Remote Sensing Laboratory, Universitè catholique de Louvain, Louvain La Neuve, Belgium and Center for Advanced Communications, Villanova University, Villanova, PA, USA, from 2006 to 2010 and 2010 to 2013, respectively. Currently, he holds a Research Engineer position at Arraycomm, LLC, San Jose, CA, USA. His research interests include cooperative and opportunistic wireless communications, robust algorithms for multi-antenna systems, convex optimization, UAV networks, and passive radar systems.

Dr. Chalise is an Associate Editor of EURASIP Journal of Communications and Networking.

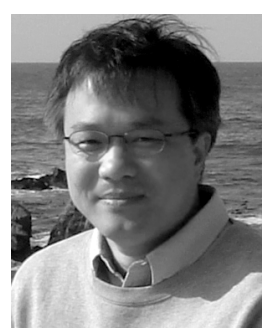

Yimin D. Zhang (SM’01) received the Ph.D. degree from the University of Tsukuba, Tsukuba, Japan, in 1988.

He joined the faculty of the Department of Radio Engineering, Southeast University, Nanjing, China, in 1988. He served as a Technical Manager at the Communication Laboratory Japan, Kawasaki, Japan, from 1995 to 1997, and was a Visiting Researcher at ATR Adaptive Communications Research Laboratories, Kyoto, Japan, from 1997 to 1998. Since 1998, he has been with the Villanova University, Villanova, PA, where he is currently a Research Professor with the Center for Advanced Communications and the Director of the Wireless Communications and Positioning Laboratory. He has more than 200 publications in the area of statistical signal and array processing for communications and radar applications, including wireless networks, cooperative communications, MIMO systems, source localization and target tracking, over-the-horizon radar, passive sensing, jammer suppression, time-frequency analysis, radio frequency identification (RFID), and image processing.

Dr. Zhang is an Associate Editor for the Journal of the Franklin Institute and an Editor for the Signal Processing journal. He is an Associate Editor for the IEEE TRANSACTIONS ON SigNAL PROCESSING and was an Associate Editor for the IEEE Signal Processing LetTers from 2006 to 2010.

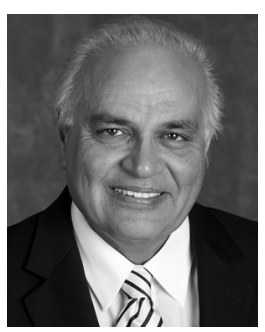

Moeness G. Amin (F'01) received the Ph.D. degree in electrical engineering in 1984 from the University of Colorado.

He has been on the Faculty of the Department of Electrical and Computer Engineering at Villanova University since 1985 . In 2002, he became the Director of the Center for Advanced Communications, College of Engineering. He has more than 450 journal and conference publications in the areas of wireless communications, time-frequency analysis, smart antennas, waveform design and diversity, interference cancellation in broadband communication platforms, anti-jam GPS, target localization and tracking, direction finding, channel diversity and equalization, ultrasound imaging, and radar signal processing.

Dr. Amin is the Recipient of the 2009 Individual Technical Achievement Award from the European Association of Signal Processing, and the Recipient of the 2010 NATO Scientific Achievement Award. He is a Fellow of the International Society of Optical Engineering, 2007; and a Fellow of the Institute of Engineering and Technology (IET), 2010. He is a Recipient of the IEEE Third Millennium Medal, 2000; Recipient of the Chief of Naval Research Challenge Award, 2010; Distinguished Lecturer of the IEEE Signal Processing Society, 2003-2004; Active Member of the Franklin Institute Committee on Science and the Arts; Recipient Villanova University Outstanding Faculty Research Award, 1997; and the Recipient of the IEEE Philadelphia Section Award, 1997. He is a member of SPIE, EURASIP, ION, Eta Kappa Nu, Sigma Xi, and Phi Kappa Phi. He currently serves on the Overview Board of the IEEE TRANSACTIONS ON SIGNAL PROCESSING. He also serves on the Editorial Board of the EURASIP Signal Processing Journal. He was a Plenary Speaker at ICASSP 2010. He was the Special Session Co-Chair of the 2008 IEEE International Conference on Acoustics, Speech, and Signal Processing. He was the Technical Program Chair of the 2nd IEEE International Symposium on Signal Processing and Information Technology, 2002. He was the General and Organization Chair of the IEEE Workshop on Statistical Signal and Array Processing, 2000. He was the General and Organization Chair of the IEEE International Symposium on Time-Frequency and Time-Scale Analysis, 1994. He was an Associate Editor of the IEEE TRANSACTIONS ON Signal PROCESSING during 1996-1998. He was a member of the IEEE Signal Processing Society Technical Committee on Signal Processing for Communications during 1998-2002. He was a Member of the IEEE Signal Processing Society Technical Committee on Statistical Signal and Array Processing during 1995-1997. He has given several keynote and plenary talks, and served as a Session Chair in several technical meetings. He was the Guest Editor of the Journal of Franklin Institute (September 2008 Special Issue on Advances in Indoor Radar Imaging). He was a Guest Editor of the IEEE TRANSACtions on GeoscienCE AND REMOte SENSING (May 2009 Special Issue on Remote Sensing of Building Interior) and a Guest Editor of the ET Signal Processing (December 2009 Special Issue on Time-Frequency Approach to Radar Detection, Imaging, and Classification). 